\title{
Restricted Jacobi Fields
}

\author{
Dennis I. Barrett and Claudiu C. Remsing* \\ (Communicated by Josef Mikes)
}

\begin{abstract}
We generalize the concept of a Jacobi field to nonholonomic Riemannian geometry, considering both nonholonomic Jacobi fields, and, more generally, restricted Jacobi fields. In the first case, the corresponding Jacobi equation involves the nonholonomic connection and the Schouten curvature tensor. In the second case, the Jacobi equation involves connections and curvature tensors arising in the construction of the Wagner curvature tensor. We also briefly discuss the existence of restricted Jacobi fields.
\end{abstract}

Keywords: Nonholonomic Riemannian structure, Jacobi field, Schouten curvature tensor, Wagner curvature tensor.

AMS Subject Classification (2020): Primary: 70G45; Secondary: 37J60.

\section{Introduction}

Riemannian geometry admits two natural generalizations. One generalization-sub-Riemannian geometryis essentially the study of the shortest admissible curves, where "admissible" means "tangent to a non-integrable distribution D." (A fundamental concept in sub-Riemannian geometry is the Carnot-Carathéodory distance; the normal sub-Riemannian geodesics are precisely the local length-minimizers of this distance.) Another generalization-nonholonomic Riemannian geometry-deals with the straightest admissible curves, where "straightest" refers to the curves whose acceleration (specified by means of a connection) vanishes. (The nonholonomic geodesics are precisely the geodesics of a distinguished connection, called the nonholonomic connection; this connection is pre-eminent in nonholonomic Riemannian geometry.) We note that, in the past, the term "nonholonomic Riemannian geometry" was used to refer to both of the above-mentioned geometries. However, we shall use it to refer exclusively to the second; the term "sub-Riemannian geometry" has now also become fairly standard.

Sub-Riemannian geometry has seen a surge of recent activity (see, e.g., $[9,19,20,1]$ and references therein); by contrast, there has been relatively little recent effort devoted to nonholonomic Riemannian geometry. The initial development of nonholonomic Riemannian geometry started in the first half of the last century, involving geometers such as E. Cartan, Vrănceanu, Synge, Schouten, and Wagner. More recent efforts, however, have primarily been from the perspective of nonholonomic mechanical systems. (A nonholonomic Riemannian manifold models the motion of a particle with linear-in-velocities nonholonomic constraints and a kinetic-energy Lagrangian.) Some textbooks on nonholonomic Riemannian geometry (particularly, from the perspective of nonholonomic mechanics) are [10, 12]; a few notable modern papers (following a more geometric approach) are $[22,23,17,13,15,14,21]$ (see also $[8,6,7,5]$ ).

Jacobi fields are effective instruments for studying Riemannian manifolds. Such fields are the variation vector fields of one-parameter (geodesic) variations of Riemannian geodesics. They provide insights into the behaviour of geodesics (e.g., they appear in both the first and second variation formulas), as well as the curvature of the manifold (the Riemannian curvature controls the infinitesimal spreading of the family of geodesics). They also find use in establishing comparison theorems. Jacobi fields have been generalized to sub-Riemannian geometry (or, more generally, geometric optimal control theory) [2,3], and used, in particular, to define the curvature of a sub-Riemannian manifold (see [4] and references therein). 
In this paper Jacobi fields are generalized to nonholonomic Riemannian geometry. First, we consider variations not of nonholonomic geodesics, but of their tangent lifts, and characterize their variation vector fields. Second, we project these variation vector fields back to the base manifold, and hence derive the analogue of the Jacobi equation (i.e., a second-order ordinary differential equation describing the infinitesimal behaviour of the Jacobi fields). The paper is organized as follows. Section 2 covers necessary prerequisites from nonholonomic Riemannian geometry. In particular, we recall the concept of a restricted connection, the definition of a nonholonomic Riemannian structure and the nonholonomic connection, and the Schouten and Wagner curvature tensors. The concept of a nonholonomic variation, and (more generally) a restricted variation, of a nonholonomic geodesic, are introduced. Section 3 and Section 4 contain the main contributions of the paper, wherein we consider nonholonomic Jacobi fields and restricted Jacobi fields, respectively. In both sections, we derive the Jacobi equation. For the case of a nonholonomic Jacobi field (i.e., a Jacobi field that corresponds to a nonholonomic variation), this involves (a component of) the Schouten curvature tensor. For the case of a restricted Jacobi field, the Jacobi equation involves (components of) curvature tensors arising in the construction of the Wagner curvature tensor. (In particular, while nonholonomic Jacobi fields are defined on any nonholonomic Riemannian manifold, the restricted Jacobi fields may only be defined for structures on which the Wagner tensor may be defined.) Lastly, in Section 5, the existence of restricted Jacobi fields is briefly discussed.

\section{Preliminaries}

We shall adhere to the following conventions in this paper. All manifolds, vector fields, etc. under consideration are assumed to be smooth, i.e., of class $\mathcal{C}^{\infty}$. All distributions are assumed to be regular, i.e., to have constant rank. We shall also use the summation convention on repeated indices. Unless stated otherwise, we shall assume that $a, b, c$ range through $1, \ldots, r$; that $a_{i}, b_{i}, c_{i}$ range through $1, \ldots, r_{i}$; and that $i$ ranges through $1, \ldots, N-1$.

\subsection{Restricted connections}

Let $\mathrm{M}$ be a (finite-dimensional) manifold, and let $\mathcal{E}$ and $\mathcal{D}$ be distributions on $\mathrm{M}$. We shall assume that any two points in $\mathrm{M}$ can be joined by a $\mathcal{E}$-curve (i.e., a curve tangent to $\mathcal{E}$ ). An $\mathcal{E}$-restricted connection on $\mathcal{D}$ (or simply an $\mathcal{E}$-connection on $\mathcal{D}$ ) is a mapping $\nabla: \Gamma(\mathcal{E}) \times \Gamma(\mathcal{D}) \rightarrow \Gamma(\mathcal{D})$ such that

$$
\nabla_{f X} U=f \nabla_{X} U \text { and } \nabla_{X}(f U)=X[f] U+f \nabla_{X} U
$$

for $f \in \mathcal{C}^{\infty}(\mathrm{M}), X \in \Gamma(\mathcal{E})$, and $U \in \Gamma(\mathcal{D})$. The expression $\nabla_{X} U(q), q \in \mathrm{M}$ depends only on the value of $X$ at $q$, and the value of $U$ along any $\mathcal{E}$-curve tangent to $X(q)$.

Let $\nabla$ be an $\mathcal{E}$-connection on $\mathcal{D}$, and let $\gamma:[0, T] \rightarrow$ M be an $\mathcal{E}$-curve. A section $V \in \Gamma\left(\gamma^{*} \mathcal{D}\right)$ (where $\Gamma\left(\gamma^{*} \mathcal{D}\right)$ denotes the space of sections of $\mathcal{D}$ along $\gamma$ ) is called parallel along $\gamma$ if $\nabla_{\dot{\gamma}} V=0$. A vector field $U \in \Gamma(\mathcal{D})$ is called parallel if $\nabla U=0$. ( $U$ is parallel if and only if $U \circ \gamma$ is parallel along $\gamma$ for every $\mathcal{E}$-curve $\gamma$.)

Proposition 2.1. Let $V_{0} \in \mathcal{D}_{\gamma(0)}$; there exists a unique section $V \in \Gamma\left(\gamma^{*} \mathcal{D}\right)$ such that $V(0)=V_{0}$. ( $V$ is called the parallel translate of $V_{0}$ along $\gamma$.)

The parallel translation along $\gamma$ is the linear map $\Pi_{\gamma}^{t}: \mathcal{D}_{\gamma(0)} \rightarrow \mathcal{D}_{\gamma(t)}, t \in[0, T]$ defined as $\Pi_{\gamma}^{t}\left(V_{0}\right)=V(t)$, where $V$ is the parallel translate of $V_{0}$ along $\gamma$.

Let $\pi: \mathcal{D} \rightarrow \mathrm{M}$ and $\tau: T \mathcal{D} \rightarrow \mathcal{D}$ be the natural projections, and let $\mathcal{V}=\operatorname{ker} T \pi$ be the vertical distribution. The map $v: \pi^{*} \mathcal{D} \rightarrow \mathcal{V}$ given by

$$
v\left(U_{q}, V_{q}\right)=\left.\frac{d}{d t}\right|_{t=0}\left(U_{q}+t V_{q}\right), \quad\left(U_{q}, V_{q}\right) \in \pi^{*} \mathcal{D}
$$

is a vector bundle isomorphism. If $U_{q} \in \mathcal{D}$, then the linear isomorphism $v_{U_{q}}=v\left(U_{q}, \cdot\right): \mathcal{D}_{q} \rightarrow \mathcal{V}_{U_{q}}$ is called the vertical lift over $U_{q}$. The vertical lift of $X \in \Gamma(\mathcal{D})$ is the vector field $X^{v} \in \Gamma(\mathcal{V})$ given by

$$
X^{v}\left(U_{q}\right)=v\left(U_{q}, X(q)\right), \quad U_{q} \in \mathcal{D} .
$$

The pullback bundle $\pi^{*} \mathcal{E}$ admits two bundle structures, viz., $\varpi_{1}: \pi^{*} \mathcal{E} \ni\left(U_{q}, X_{q}\right) \mapsto U_{q} \in \mathcal{D}$ and $\varpi_{2}: \pi^{*} \mathcal{E} \ni$ $\left(U_{q}, X_{q}\right) \mapsto X_{q} \in \mathcal{E}$. The restricted connection $\nabla$ induces a mapping $h: \pi^{*} \mathcal{E} \rightarrow T \mathcal{D}$, defined as

$$
h\left(U_{q}, X_{q}\right)=T_{q} U \cdot X_{q}-v\left(U_{q}, \nabla_{X_{q}} U\right), \quad\left(U_{q}, X_{q}\right) \in \pi^{*} \mathcal{E} .
$$

Here $U \in \Gamma(\mathcal{D})$ satisfies $U(q)=U_{q}$; the definition of $h$ does not depend on the choice of $U$. The map $h$ is: 
1. a linear bundle map from $\varpi_{1}$ to $\tau$ covering the identity, i.e., $\tau \circ h=\varpi_{1}$;

2. a bundle map from $\varpi_{2}$ to $T \pi$ covering the inclusion $\iota: \mathcal{E} \rightarrow T \mathrm{M}$, i.e., $T \pi \cdot h=i \circ \varpi_{2}$;

3. linear, i.e., $T_{U_{q}} \phi_{t} \cdot h\left(U_{q}, X_{q}\right)=h\left(\phi_{t}\left(U_{q}\right), X_{q}\right)$ for every $\left(U_{q}, X_{q}\right) \in \pi^{*} \mathcal{E}$, where $\phi_{t}: \mathcal{D} \rightarrow \mathcal{D}, U_{q} \mapsto e^{t} U_{q}$ is the canonical dilation.

Let $\mathcal{H}=\operatorname{im} h$ be the horizontal distribution; then $\pi_{*} \mathcal{H}=\mathcal{E}, \mathcal{V} \cap \mathcal{H}=\{0\}$, and $V+\mathcal{H} \subseteq T \mathcal{D}$ with equality if and only if $\mathcal{E}=T \mathrm{M}$. If $U_{q} \in \mathcal{D}$, then the linear isomorphism $h\left(U_{q}, \cdot\right): \mathcal{E}_{q} \rightarrow \mathcal{H}_{U_{q}}$ is called the horizontal lift (or $h$-lift) over $U_{q}$. The horizontal lift (or $h$-lift) of $X \in \Gamma(\mathcal{E})$ is the vector field $X^{h} \in \Gamma(\mathcal{H})$ given by

$$
X^{h}\left(U_{q}\right)=h\left(U_{q}, X(q)\right), \quad U_{q} \in \mathcal{D} .
$$

Projectable horizontal vector fields (i.e., vector fields $X \in \Gamma(\mathcal{H})$ for which there exists $Y \in \Gamma(T \mathrm{M})$ such that $T \pi \cdot X=Y \circ \pi)$ are precisely the horizontal lifts of vector fields in $\Gamma(\mathcal{E})$. It should be clear that the Lie bracket of any vector field with a vertical vector field is again vertical. In particular, if $X \in \Gamma(\mathcal{E})$ and $U \in \Gamma(\mathcal{D})$, then $\left[X^{v}, U^{h}\right]=\left(\nabla_{X} U\right)^{v}$. In general, the bracket of two horizontal vector fields will not be horizontal (or even be a section of $\mathcal{V} \oplus \mathcal{H})$.

Lastly, we mention the following useful equations. If $X \in \Gamma(\mathcal{D}), Y \in \Gamma(\mathcal{E})$, and $\omega \in \Gamma\left(\mathcal{D}^{*}\right)$, then

$$
X^{v}[\bar{\omega}]=\langle\omega, X\rangle \circ \pi \quad \text { and } \quad Y^{h}[\bar{\omega}]=\overline{\nabla_{Y} \omega} .
$$

Here $\bar{\omega} \in \mathcal{C}^{\infty}(\mathcal{D})$ the function given by $\bar{\omega}\left(U_{q}\right)=\left\langle\omega_{q}, U_{q}\right\rangle$, and $\langle\cdot, \cdot\rangle: \mathcal{D}^{*} \otimes \mathcal{D} \rightarrow \mathbb{R}$ is the canonical pairing.

Remark 2.1. The concept of a restricted connection (as a covariant derivative) was first introduced in [15]; such connections (particularly, viewed as a horizontal lift) are also essentially covered in [11]. For more explicit details on restricted connections (particularly, in the context of nonholonomic Riemannian geometry) see [7].

\subsection{Nonholonomic Riemannian structures}

A distribution $\mathcal{D}$ on a manifold $\mathrm{M}$ is said to be completely nonholonomic if there exists $N \geq 2$ (called the degree of nonholonomy of $\mathcal{D}$ ) such that $\mathcal{D}^{1} \subsetneq \cdots \subsetneq \mathcal{D}^{N-1} \subsetneq \mathcal{D}^{N}=T \mathrm{M}$, where $\mathcal{D}^{1}=\mathcal{D}$ and $\mathcal{D}^{i+1}=\mathcal{D}^{i}+\left[\mathcal{D}^{i}, \mathcal{D}^{i}\right]$ for $i \geq 1$. (Each distribution $\mathcal{D}^{i+1}$ is also assumed to be regular, since this is not implied by the regularity of $\mathcal{D}$.) If $\mathcal{D}$ is completely nonholonomic and $N=2$, then $\mathcal{D}$ is called strongly nonholonomic. Given a distribution $\mathcal{D}$ and a curve $\gamma:[0, T] \rightarrow \mathrm{M}$, the space of all vector fields along $\gamma$ is naturally identified with the space $\Gamma\left(\gamma^{*} \mathcal{D}\right)$ of sections of the pullback bundle $\gamma^{*} \mathcal{D}$. The curve $\gamma$ is called a $\mathcal{D}$-curve if $\dot{\gamma} \in \Gamma\left(\gamma^{*} \mathcal{D}\right)$; the Chow-Rashevskii theorem asserts that completely nonholonomy of $\mathcal{D}$ is a sufficient condition for any two points in $\mathrm{M}$ to be joined by a (piecewise) $\mathcal{D}$-curve.

A nonholonomic Riemannian manifold (or nonholonomic Riemannian structure) is a quadruple $\left(\mathrm{M}, \mathcal{D}, \mathcal{D}^{\perp}, \mathrm{g}\right.$ ), where $\mathrm{M}$ is an $n$-dimensional manifold, $\mathcal{D}$ is a rank $r<n$ completely nonholonomic distribution on $\mathrm{M}, \mathcal{D}^{\perp}$ is a distribution complementary to $\mathcal{D}$ (i.e., $T \mathrm{M}=\mathcal{D} \oplus \mathcal{D}^{\perp}$ ), and $\mathbf{g}$ is a (positive definite) metric tensor defined on elements of $\mathcal{D}$. Let $\mathscr{P}$ denote the projection onto $\mathcal{D}$ along $\mathcal{D}^{\perp}$, and let $\mathscr{Q}$ be the complementary projection onto $\mathcal{D}^{\perp}$. The projected Lie bracket $\mathscr{P}([\cdot, \cdot]): \Gamma(T \mathrm{M}) \times \Gamma(T \mathrm{M}) \rightarrow \Gamma(\mathcal{D})$ will be denoted by $\llbracket \cdot, \cdot \rrbracket$. If $Z \in \Gamma(T \mathrm{M})$, then we denote by $£_{Z}$ the Lie derivative along $Z$, and by $£_{Z}^{\mathscr{P}}$ the derivation given by $£_{Z}^{\mathscr{P}} f=Z[f], £_{Z}^{\mathscr{P}} X=\llbracket Z, X \rrbracket$ for $f \in \mathcal{C}^{\infty}(\mathrm{M})$ and $X \in \Gamma(\mathcal{D})$. ( $£_{Z}^{\mathscr{P}}$ is a restricted tensor derivation; see [7].) Similarly, if $\gamma$ is a $\mathcal{D}$-curve, then we shall denote by $£_{\dot{\gamma}}^{\mathscr{Q}}$ the mapping

$$
\Gamma\left(\gamma^{*} T \mathrm{M}\right) \rightarrow \Gamma\left(\gamma^{*} \mathcal{D}^{\perp}\right), \quad J \mapsto £_{\dot{\gamma}}^{\mathscr{Q}} J=\mathscr{Q}([Z, \widetilde{J}]) \circ \gamma,
$$

where $Z \in \Gamma(\mathcal{D})$ and $\widetilde{J} \in \Gamma(T \mathrm{M})$ are extensions of $\dot{\gamma}$ and $J$ to an open neighbourhood of $\gamma$, respectively. (One may verify that $£_{\dot{\gamma}}^{\mathscr{Q}} J$ does not depend on the choice of extensions $Z$ and $\widetilde{J}$.)

Let $\left(\mathrm{M}, \mathcal{D}, \mathcal{D}^{\perp}, \mathrm{g}\right)$ be a nonholonomic Riemannian manifold. Associated to $\left(\mathrm{M}, \mathcal{D}, \mathcal{D}^{\perp}, \mathrm{g}\right)$ is a restricted connection $\nabla$, called the nonholonomic connection. It is the unique connection $\nabla: \Gamma(\mathcal{D}) \times \Gamma(\mathcal{D}) \rightarrow \Gamma(\mathcal{D})$ that is both metric and torsion free, i.e.,

$$
Z[\mathbf{g}(X, Y)]=\mathbf{g}\left(\nabla_{Z} X, Y\right)+\mathbf{g}\left(X, \nabla_{Z} Y\right) \quad \text { and } \quad \nabla_{X} Y-\nabla_{Y} X=\llbracket X, Y \rrbracket
$$

for every $X, Y, Z \in \Gamma(\mathcal{D})$ (see, e.g., [16] for a proof of this fact). (The torsion of $\nabla$ is the tensor $\mathcal{D} \wedge \mathcal{D} \rightarrow \mathcal{D}$, $X \wedge Y \mapsto \nabla_{X} Y-\nabla_{Y} X-\llbracket X, Y \rrbracket$.) Let $\gamma:[0, T] \rightarrow$ M be a $\mathcal{D}$-curve. The parallel translation along $\gamma$ (induced by the nonholonomic connection) is denoted by $\Pi_{\gamma}^{t}$. Furthermore, $\gamma$ is called a nonholonomic geodesic if $\nabla_{\dot{\gamma}} \dot{\gamma}(t)=0$ 
for every $t \in[0, T]$. In this paper, we shall assume that all nonholonomic geodesics under consideration are regular, i.e., non-constant.

The Schouten curvature tensor is the tensor $K: \mathcal{D} \wedge \mathcal{D} \otimes \mathcal{D} \rightarrow \mathcal{D}$ given by

$$
K(X \wedge Y) Z=\left[\nabla_{X}, \nabla_{Y}\right] Z-\nabla_{\llbracket X, Y \rrbracket} Z-£_{\mathscr{Q}([X, Y])}^{\mathscr{P}} Z, \quad X, Y, Z \in \Gamma(\mathcal{D}) .
$$

Let $\widehat{K}(W, X, Y, Z)=\mathbf{g}(K(W \wedge X) Y, Z)$ for $W, X, Y, Z \in \Gamma(\mathcal{D})$, and let

$$
\widehat{R}(W, X, Y, Z)=\frac{1}{2}(\widehat{K}(W, X, Y, Z)-\widehat{K}(W, X, Z, Y)), \quad \widehat{C}=\widehat{K}-\widehat{R} .
$$

We have the following symmetries:

S1. $\widehat{K}(W, X, Y, Z)+\widehat{K}(X, W, Y, Z)=0$;

S2. $\widehat{K}(W, X, Y, Z)+\widehat{K}(X, Y, W, Z)+\widehat{K}(Y, W, X, Z)=0$;

S3. $\widehat{R}(W, X, Y, Z)+\widehat{R}(W, X, Z, Y)=0$;

S4. $\widehat{R}(W, X, Y, Z)=\widehat{R}(Y, Z, W, X)$;

S5. $\widehat{C}(W, X, Y, Z)=\widehat{C}(W, X, Z, Y)$.

$\widehat{R}$ satisfies all the symmetries of the corresponding Riemannian tensor; hence it may be thought of as the "Riemannian" component of $\widehat{K}$, whereas $\widehat{C}$ is a "non-Riemannian remainder." It turns out that $\widehat{C}$ may be expressed quite simply, in terms of the metric and the projection operators:

$$
\widehat{C}(W, X, Y, Z)=\frac{1}{2}\left(£_{\mathscr{Q}([W, X])}^{\mathscr{g}} \mathbf{g}\right)(Y, Z) .
$$

Let $R$ and $C$ be the tensors defined implicitly by the equations $\widehat{R}(W, X, Y, Z)=\mathbf{g}(R(W \wedge X) Y, Z)$ and $\widehat{C}(W, X, Y, Z)=\mathbf{g}(C(W \wedge X) Y, Z)$; clearly, we have $K=R+C$. For each $U \in \Gamma(\mathcal{D})$, the Jacobi operator $\Psi_{U}$ (cf. [18]) is the tensor $\Psi_{U}(X)=R(X \wedge U) U$ for $X \in \Gamma(\mathcal{D})$. By the symmetries of $\widehat{R}$, we have that $\Psi_{U}$ is selfadjoint; furthermore, the curvature tensor $R$ is completely determined by the (family of) Jacobi operators $\left\{\Psi_{U}: U \in \Gamma(\mathcal{D})\right\}$.

The nonholonomic connection may be canonically extended to a vector bundle connection $\nabla^{\circ}$ on $\mathcal{D}$ as follows:

$$
\stackrel{\circ}{\nabla}: \Gamma(T \mathrm{M}) \times \Gamma(\mathcal{D}) \rightarrow \Gamma(\mathcal{D}), \quad \stackrel{\circ}{\nabla}_{Z}=\nabla_{\mathscr{P}(Z)}+£_{\mathscr{Q}(Z)}^{\mathscr{P}} .
$$

Let $\pi: \mathcal{D} \rightarrow \mathrm{M}$ be the natural projection. Associated to $\nabla$ and $\nabla^{\circ}$, respectively, are the restricted connections $h: \pi^{*} \mathcal{D} \rightarrow T \mathcal{D}$ and $f: \pi^{*} T \mathrm{M} \rightarrow T \mathcal{D}$, given by

$$
h\left(U_{q}, X_{q}\right)=T_{q} U \cdot X_{q}-v\left(U_{q}, \nabla_{X_{q}} U\right) \text { and } f\left(U_{q}, Z_{q}\right)=T_{q} U \cdot Z_{q}-v\left(U_{q}, \stackrel{\circ}{\nabla}_{Z_{q}} U\right)
$$

for $\left(U_{q}, X_{q}\right) \in \pi^{*} \mathcal{D},\left(U_{q}, Z_{q}\right) \in \pi^{*} T$ M. Notice that, by definition of $\stackrel{\circ}{\nabla}$, we have $\left.f\right|_{\pi^{*} \mathcal{D}}=h$. Let $\mathcal{V}=\operatorname{ker} T \pi$ be the vertical distribution, $\mathcal{H}=\operatorname{im} h$ and $\mathcal{H}^{\perp}=\left.\operatorname{im} f\right|_{\pi^{*} \mathcal{D}^{\perp}}$; then $T \mathcal{D}=\mathcal{V} \oplus \mathcal{H} \oplus \mathcal{H}^{\perp}$. Let $\mathscr{V}: T \mathcal{D} \rightarrow \mathcal{V}, \mathscr{P}: T \mathcal{D} \rightarrow \mathcal{V} \oplus \mathcal{H}$ and $\mathscr{Q}: T \mathcal{D} \rightarrow \mathcal{H}^{\perp}$ be projections corresponding to the foregoing decomposition of $T \mathcal{D}$. We shall also denote the projected Lie bracket $\mathscr{P}([\cdot, \cdot]): \Gamma(T \mathcal{D}) \times \Gamma(T \mathcal{D}) \rightarrow \Gamma(\mathcal{V} \oplus \mathcal{H})$ by $\llbracket \cdot, \cdot]$. If $X \in \Gamma\left(\mathcal{H} \oplus \mathcal{H}^{\perp}\right)$ is projectable, then $\mathscr{P}(X)=\mathscr{P}\left(\pi_{*} X\right)^{h}$ and $\mathscr{Q}(X)=\mathscr{Q}\left(\pi_{*} X\right)^{f}$. Hence we can express the Lie bracket of two horizontally-lifted vector fields:

$$
\left[X^{h}, Y^{h}\right]=\mathscr{V}\left(\left[X^{h}, Y^{h}\right]\right)+\llbracket X, Y \rrbracket^{h}+\mathscr{Q}([X, Y])^{f}, \quad X, Y \in \Gamma(\mathcal{D}) .
$$

The vertical component $\mathscr{V}\left(\left[X^{h}, Y^{h}\right]\right)$ may be expressed in terms of the Schouten curvature tensor.

Proposition 2.2 ([7]). We have

$$
K(X \wedge Y) U_{q}=-v_{U_{q}}^{-1} \cdot \mathscr{V}\left(\llbracket X^{h}, Y^{h} \rrbracket\right)\left(U_{q}\right)
$$

for $X, Y \in \Gamma(\mathcal{D})$ and $U_{q} \in \mathcal{D}$.

Let $\Xi \in \Gamma(T \mathcal{D})$ be the vector field given by $\Xi\left(X_{q}\right)=h\left(X_{q}, X_{q}\right)$ for $X_{q} \in \mathcal{D}$; then

1. $T \pi \cdot \Xi=\iota$, where $\iota: \mathcal{D} \rightarrow T \mathrm{M}$ is the inclusion map.

2. $\Xi \circ \phi_{t}=T \phi_{t} \cdot e^{t} \Xi$, where $\phi_{t}: \mathcal{D} \rightarrow \mathcal{D}, X_{q} \mapsto e^{t} X_{q}$ is the canonical dilation.

3. If $\gamma$ is a nonholonomic geodesic, then $\dot{\gamma}$ is an integral curve of $\Xi$; conversely, if $\lambda$ is an integral curve of $\Xi$, then $\pi \circ \lambda$ is a nonholonomic geodesic.

$\Xi$ is called the nonholonomic geodesic spray; its flow $\Phi_{t}$ is called the nonholonomic geodesic flow. 


\subsection{The Wagner curvature tensor}

Let $\left(\mathrm{M}, \mathcal{D}, \mathcal{D}^{\perp}, \mathrm{g}\right)$ be a nonholonomic Riemannian structure, where $\mathcal{D}$ has rank $r_{0}$ and degree of nonholonomy $N$. Let $\mathcal{E}^{1}, \ldots, \mathcal{E}^{N-1}$ be distributions on $\mathrm{M}$ of rank $r_{1}, \ldots, r_{N-1}$, respectively, such that

$$
\mathcal{D}^{\perp}=\mathcal{E}^{1} \oplus \cdots \oplus \mathcal{E}^{N-1} \quad \text { and } \quad \mathcal{D}^{i+1}=\mathcal{D}^{i} \oplus \mathcal{E}^{i} .
$$

Generally there is no canonical choice of $\mathcal{E}^{1}, \ldots, \mathcal{E}^{N-1}$. We shall refer to a nonholonomic Riemannian structure, together with a particular choice of distributions $\mathcal{E}^{1}, \ldots, \mathcal{E}^{N-1}$, as a Wagner structure. (The definition of the Wagner curvature tensor depends on the choice of $\mathcal{E}^{1}, \ldots, \mathcal{E}^{N-1}$.) However, if $N=2$ (i.e., $\mathcal{D}$ is strongly nonholonomic), then there is a canonical choice, viz., $\mathcal{E}^{1}=\mathcal{D}^{\perp}$. Likewise, if $\mathrm{g}$ is the restriction to $\mathcal{D}$ of a Riemannian metric (as is the case, for instance, of a nonholonomic mechanical system with linear constraints and kinetic-energy Lagrangian), then $\mathcal{E}^{1}, \ldots, \mathcal{E}^{N-1}$ may be chosen canonically.

Let $\mathscr{Q}_{i}: T \mathrm{M} \rightarrow \mathcal{E}^{i}$ be the projection onto $\mathcal{E}^{i}$, let $\mathscr{P}_{1}=\mathscr{P}$, and let $\mathscr{P}_{i+1}: T \mathrm{M} \rightarrow \mathcal{D}^{i+1}$ be the projection onto $\mathcal{D}^{i+1}=\mathcal{D} \oplus \mathcal{E}^{1} \oplus \cdots \oplus \mathcal{E}^{i}$ given by $\mathscr{P}_{i+1}=\mathscr{P} \oplus \mathscr{Q}_{1} \oplus \cdots \oplus \mathscr{Q}_{i}$. If $\gamma$ is a $\mathcal{D}$-curve, then we shall denote by $£_{\dot{\gamma}}^{\mathscr{Q}_{i}}$ the mapping

$$
\Gamma\left(\gamma^{*} T \mathrm{M}\right) \rightarrow \Gamma\left(\gamma^{*} \mathcal{E}^{i}\right), \quad J \mapsto £_{\dot{\gamma}}^{\mathscr{Q}_{i}} J=\mathscr{Q}_{i}([Z, \widetilde{J}]) \circ \gamma,
$$

where $Z \in \Gamma(\mathcal{D})$ and $\widetilde{J} \in \Gamma\left(\mathcal{D}^{k}\right)$ are extensions of $\dot{\gamma}$ and $J$ to an open neighbourhood of $\gamma$, respectively. (One may verify that $£_{\dot{\gamma}}^{\mathscr{Q}_{i}} J$ does not depend on the choice of extensions $Z$ and $\widetilde{J}$.)

Let $\Lambda_{i}: \mathcal{D}^{i} \wedge \mathcal{D}^{i} \rightarrow \mathcal{E}^{i}$ be the tensor given by $\Lambda_{i}(X \wedge Y)=\mathscr{Q}_{i}([X, Y])$ for $X, Y \in \Gamma\left(\mathcal{D}^{i}\right)$. Since $\mathcal{D}$ is completely nonholonomic, we have that each map $\Lambda_{i}$ is surjective; hence g may be extended to a Riemannian metric on $M$.

Proposition 2.3 (cf. [7], [13]). There exists a unique Riemannian metric $\widetilde{\mathbf{g}}$ on $\mathrm{M}$ satisfying the following conditions:

1. The decomposition $T \mathbf{M}=\mathcal{D} \oplus \mathcal{E}^{1} \oplus \cdots \oplus \mathcal{E}^{N-1}$ is orthogonal and $\widetilde{\mathbf{g}}=\mathbf{g} \oplus \mathbf{h}^{1} \oplus \cdots \oplus \mathbf{h}^{N-1}$, where $\mathbf{h}^{i}=\left.\widetilde{\mathbf{g}}\right|_{\mathcal{E}^{i}}$.

2. Each map $\left.\Lambda_{i}\right|_{\left(\operatorname{ker} \Lambda_{i}\right)^{\perp}}:\left(\operatorname{ker} \Lambda_{i}\right)^{\perp} \rightarrow \mathcal{E}^{i}$ satisfies

$$
\mathbf{h}^{i}\left(\Lambda_{i}(W \wedge X), \Lambda_{i}(Y \wedge Z)\right)=\widehat{\mathbf{g}}^{i}(W \wedge X, Y \wedge Z)
$$

for $W \wedge X, Y \wedge Z \in\left(\operatorname{ker} \Lambda_{i}\right)^{\perp}$. Here $\widehat{\mathbf{g}}^{i}$ is the metric induced on $\mathcal{D}^{i} \wedge \mathcal{D}^{i}$ by the metric $\mathbf{g}^{i}=\mathbf{g} \oplus \mathbf{h}^{1} \oplus \cdots \oplus \mathbf{h}^{i-1}$ on $\mathcal{D}^{i}$, i.e., $\widehat{\mathbf{g}}^{i}(W \wedge X, Y \wedge Z)=\mathbf{g}^{i}(W, Y) \mathbf{g}^{i}(X, Z)-\mathbf{g}^{i}(W, Z) \mathbf{g}^{i}(X, Y)$, and $\left(\operatorname{ker} \Lambda_{i}\right)^{\perp}$ is the orthogonal complement of $\operatorname{ker} \Lambda_{i}$.

Let $\nabla^{1}=\nabla$, and let $\nabla^{i+1}: \Gamma\left(\mathcal{D}^{i+1}\right) \times \Gamma(\mathcal{D}) \rightarrow \Gamma(\mathcal{D})$ be the restricted connection given by

$$
\nabla_{X}^{i+1} U=\nabla_{\mathscr{P}_{i}(X)}^{i} U+K^{i}\left(\Theta_{i}\left(\mathscr{Q}_{i}(X)\right)\right) U+£_{\mathscr{Q}_{i}(X)}^{\mathscr{P}} U
$$

for $X \in \Gamma\left(\mathcal{D}^{i+1}\right), U \in \Gamma(\mathcal{D})$. Here $\Theta_{i}=\left.\Lambda_{i}\right|_{\left(\operatorname{ker} \Lambda_{i}\right)^{\perp}} ^{-1}: \mathcal{E}^{i} \rightarrow\left(\operatorname{ker} \Lambda_{i}\right)^{\perp}$, and $K^{i}: \mathcal{D}^{i} \wedge \mathcal{D}^{i} \otimes \mathcal{D} \rightarrow \mathcal{D}$ is the curvature tensor

$$
K^{i}(X \wedge Y) U=\left[\nabla_{X}^{i}, \nabla_{Y}^{i}\right] U-\nabla_{\mathscr{P}_{i}([X, Y])}^{i} U-£_{\mathscr{Q}_{i}([X, Y])}^{\mathscr{P}} U
$$

for $X, Y \in \Gamma\left(\mathcal{D}^{i}\right), U \in \Gamma(\mathcal{D})$. Notice that $\nabla^{N}$ is a vector bundle connection on $\mathcal{D}$; its curvature tensor $K^{N}$ is called the Wagner curvature tensor.

Lemma 2.1. If $X \in \Gamma\left(\mathcal{D}^{i+1}\right)$, then $\nabla_{X}^{i+1}=\stackrel{\circ}{\nabla}_{X}+\sum_{j=1}^{i} K^{j}\left(\Theta_{j}\left(\mathscr{Q}_{j}(X)\right)\right)$.

Proof. We use induction on $i$. Consider the case $i=1$. For $X \in \Gamma\left(\mathcal{D}^{2}\right)$, we have $\mathscr{P}(X)=\mathscr{P}_{1}(X)$ and $\mathscr{Q}(X)=$ $\mathscr{Q}_{1}(X)$. Hence

$$
\nabla_{X}^{2}=\nabla_{\mathscr{P}(X)}+K^{1}\left(\Theta_{1}\left(\mathscr{Q}_{1}(X)\right)\right)+\mathfrak{E}_{\mathscr{Q}_{1}(X)}^{\mathscr{P}}=\stackrel{\circ}{\nabla}_{X}+K^{1}\left(\Theta_{1}\left(\mathscr{Q}_{1}(X)\right)\right) .
$$


Suppose now that the result holds for some $1 \leq i<N-1$ and let $X \in \Gamma\left(\mathcal{D}^{i+2}\right)$; then

$$
\begin{aligned}
\nabla_{X}^{i+2}= & \nabla_{\mathscr{P}_{i+1}(X)}^{i+1}+K^{i+1}\left(\Theta_{i+1}\left(\mathscr{Q}_{i+1}(X)\right)\right)+£_{\mathscr{Q}_{i+1}(X)}^{\mathscr{P}_{i+1}} \\
= & \nabla_{\mathscr{P}\left(\mathscr{P}_{i+1}(X)\right)}+\sum_{j=1}^{i} K^{j}\left(\Theta_{j}\left(\mathscr{Q}_{j}\left(\mathscr{P}_{i+1}(X)\right)\right)+£_{\mathscr{Q}\left(\mathscr{P}_{i+1}(X)\right)}^{\mathscr{P}}\right. \\
& \quad+K^{i+1}\left(\Theta_{i+1}\left(\mathscr{Q}_{i+1}(X)\right)\right)+£_{\mathscr{Q}_{i+1}(X)}^{\mathscr{P}} \\
= & \nabla_{\mathscr{P}(X)}+\sum_{j=1}^{i+1} K^{j}\left(\Theta_{j}\left(\mathscr{Q}_{j}(X)\right)\right)+£_{\mathscr{Q}(X)}^{\mathscr{P}} \\
= & \nabla_{X}+\sum_{j=1}^{i+1} K^{j}\left(\Theta_{j}\left(\mathscr{Q}_{j}(X)\right)\right),
\end{aligned}
$$

which proves the inductive hypothesis.

Let $\widehat{K}^{1}=\widehat{K}$, and $\widehat{K}^{i+1}(X, Y, U, V)=\mathbf{g}\left(K^{i}(X \wedge Y) U, V\right)$ for $X, Y \in \Gamma\left(\mathcal{D}^{i+1}\right), U, V \in \Gamma(\mathcal{D})$. Let $\widehat{R}^{1}=\widehat{R}, \widehat{C}^{1}=\widehat{C}$,

$$
\widehat{R}^{i+1}(X, Y, U, V)=\frac{1}{2}\left(\widehat{K}^{i+1}(X, Y, U, V)-\widehat{K}^{i+1}(X, Y, V, U)\right)
$$

and $\widehat{C}^{i+1}=\widehat{K}^{i+1}-\widehat{R}^{i+1}$. We have

$$
\widehat{C}^{i+1}(X, Y, U, V)=\frac{1}{2}\left(£_{\mathscr{Q}_{i}([X, Y])}^{\mathscr{P}}\right)(U, V) .
$$

Let $R^{1}=R, C^{1}=C$, and let $R^{i+1}$ and $C^{i+1}$ be the tensors defined implicitly by the equations $\widehat{R}^{i+1}(X, Y, U, V)=$ $\mathbf{g}\left(R^{i+1}(X \wedge Y) U, V\right)$ and $\widehat{C}^{i+1}(X, Y, U, V)=\mathbf{g}\left(C^{i+1}(X \wedge Y) U, V\right)$, respectively. Clearly, we have $K^{i+1}=R^{i+1}+$ $C^{i+1}$; furthermore, $C^{N}$ vanishes identically.

Let $\pi: \mathcal{D} \rightarrow \mathrm{M}$ be the natural projection. Associated to $\nabla^{i+1}$ is the restricted connection $h^{i+1}: \pi^{*} \mathcal{D}^{i+1} \rightarrow T \mathcal{D}$, given by

$$
h^{i+1}\left(U_{q}, X_{q}\right)=T_{q} U \cdot X_{q}-v\left(U_{q}, \nabla_{X_{q}}^{i+1} U\right), \quad\left(U_{q}, X_{q}\right) \in \pi^{*} \mathcal{D}^{i+1} .
$$

We shall denote $h$ as $h^{1}$; then $\left.h^{i+1}\right|_{\pi^{*} \mathcal{D}^{i}}=h^{i}$. Let $\mathcal{V}$ be the vertical distribution, $\mathcal{H}^{1}=\mathcal{H}=\operatorname{im} h^{1}, \mathcal{H}^{i+1}=\operatorname{im} h^{i+1}$, and $\mathcal{Q}^{i}=\left.\mathrm{im} h^{i+1}\right|_{\pi^{*} \mathcal{E}}$; then $T \mathcal{D}=\mathcal{V} \oplus \mathcal{H} \oplus \mathcal{Q}^{1} \oplus \cdots \oplus \mathcal{Q}^{N-1}$ and $\mathcal{H}^{i+1}=\mathcal{H}^{i} \oplus \mathcal{Q}^{i}$. Let $\mathscr{V}: T \mathcal{D} \rightarrow \mathcal{V}, \mathscr{P}: T \mathcal{D} \rightarrow$ $\mathcal{V} \oplus \mathcal{H}$, and $\mathscr{Q}_{i}: T \mathcal{D} \rightarrow \mathcal{Q}^{i}$ be the projections corresponding to the foregoing decomposition of $T \mathcal{D}$. Let $\mathscr{P}_{1}=\mathscr{P}$ and $\mathscr{P}_{i+1}=\mathscr{P} \oplus \mathscr{Q}_{1} \oplus \cdots \oplus \mathscr{Q}_{i}$. Notice that, if $X \in \Gamma\left(\mathcal{H} \oplus \mathcal{Q}^{1} \oplus \cdots \oplus \mathcal{Q}^{N-1}\right)$ is projectable, then $\mathscr{P}_{i+1}(X)=$ $\mathscr{P}_{i+1}\left(\pi_{*} X\right)^{h^{i+1}}$ and $\mathscr{Q}_{i}(X)=\mathscr{Q}_{i}\left(\pi_{*} X\right)^{h^{i+1}}$. Hence

$$
\left[X^{h^{i+1}}, Y^{h^{i+1}}\right]=\mathscr{V}\left(\left[X^{h^{i+1}}, Y^{h^{i+1}}\right]\right)+\mathscr{P}_{i+1}([X, Y])^{h^{i+1}}+\mathscr{Q}_{i+1}([X, Y])^{h^{i+2}}
$$

for $X, Y \in \Gamma\left(\mathcal{D}^{i+1}\right)$. (If $i=N-1$, then we take $h^{N+1}=h^{N}$.) The vertical component $\mathscr{V}\left(\left[X^{h^{i+1}}, Y^{h^{i+1}}\right]\right)$ may be expressed in terms of the curvature tensor $K^{i+1}$.

Proposition 2.4 ([7]). We have

$$
K^{i+1}(X \wedge Y) U_{q}=-v_{U_{q}}^{-1} \cdot \mathscr{V}\left(\mathscr{P}_{i+1}\left(\left[X^{h^{i+1}}, Y^{h^{i+1}}\right]\right)\right)\left(U_{q}\right)
$$

for $X, Y \in \Gamma\left(\mathcal{D}^{i+1}\right)$ and $U_{q} \in \mathcal{D}$.

\subsection{Restricted variation vector fields}

Let $\left(\mathrm{M}, \mathcal{D}, \mathcal{D}^{\perp}, \mathrm{g}\right)$ be a nonholonomic Riemannian manifold and let $\mathcal{E}$ be a distribution on $\mathrm{M}$ containing $\mathcal{D}$. Let $\gamma:[0, T] \rightarrow$ M be a nonholonomic geodesic. An $\mathcal{E}$-restricted (geodesic) variation of $\gamma$ (or simply $\mathcal{E}$-variation of $\gamma$ ) is a mapping $\sigma:(-\varepsilon, \varepsilon) \times[0, T] \rightarrow \mathrm{M},(s, t) \mapsto \sigma(s, t)=\sigma^{t}(s)=\sigma_{s}(t)$ such that:

- $\sigma_{s}$ is a nonholonomic geodesic for every $s \in(-\varepsilon, \varepsilon)$, and $\sigma_{0}=\gamma$.

- the variation vector field $J$ given by $J(t)=\left.\frac{d}{d s}\right|_{s=0} \sigma_{s}(t)$ is an element of $\Gamma\left(\gamma^{*} \mathcal{E}\right)$, i.e., $J(t) \in \mathcal{E}_{\gamma(t)}$ for every $t \in[0, T]$. 
A $\mathcal{D}$-variation of $\gamma$ is simply called a nonholonomic variation of $\gamma$. Likewise, let $\mathcal{Q}$ be a distribution on $\mathcal{D}$ containing $\mathcal{H}$ and transversal to $\mathcal{V}$ (i.e., $\mathcal{V} \cap \mathcal{Q}=\{0\}$ ). A $\mathcal{Q}$-restricted (geodesic) variation of $\dot{\gamma}$ (or $\mathcal{Q}$-variation of $\dot{\gamma}$ ) is a mapping $\Sigma:(-\varepsilon, \varepsilon) \times[0, T] \rightarrow \mathcal{D},(s, t) \mapsto \Sigma(s, t)=\Sigma^{t}(s)=\Sigma_{s}(t)$ such that:

- $\Sigma_{s}$ is an integral curve of (the nonholonomic geodesic spray) $\Xi$ for every $s \in(-\varepsilon, \varepsilon)$, and $\Sigma_{0}=\dot{\gamma}$.

- the variation vector field $V$ given by $V(t)=\left.\frac{d}{d s}\right|_{s=0} \Sigma_{s}(t)$ is an element of $\Gamma\left(\dot{\gamma}^{*}(\mathcal{V} \oplus \mathcal{Q})\right)$, i.e., $V(t) \in$ $(\mathcal{V} \oplus \mathcal{Q})_{\dot{\gamma}(t)}$ for every $t \in[0, T]$.

An $\mathcal{H}$-variation of $\dot{\gamma}$ is called a nonholonomic variation of $\dot{\gamma}$.

Proposition 2.5. Let $\gamma$ be a nonholonomic geodesic.

1. If $\Sigma$ is a $\mathcal{Q}$-variation of $\dot{\gamma}$, then $\pi \circ \Sigma$ is a $\left(\pi_{*} \mathcal{Q}\right)$-variation of $\gamma$, with variation vector field $T \pi \cdot V$.

2. If $\sigma$ is an $\mathcal{E}$-variation of $\gamma$ with variation vector field $J$, then there exists a $\mathcal{Q}$-variation of $\dot{\gamma}$ whose variation vector field $V$ satisfies $T \pi \cdot V=J$ and such that $\pi_{*} \mathcal{Q}=\mathcal{E}$.

Proof. Let $\Sigma$ be a $\mathcal{Q}$-variation of $\dot{\gamma}$ and let $\sigma=\pi \circ \Sigma$. Then

$$
\sigma(s, t)=\pi(\Sigma(s, t))=\pi\left(\Phi_{t}\left(\Sigma^{0}(s)\right)\right)=\exp _{\sigma_{s}(0)}\left(t \Sigma^{0}(s)\right) .
$$

In particular, $\sigma_{s}$ is a nonholonomic geodesic for every $s \in(-\varepsilon, \varepsilon)$ and $\sigma_{0}(t)=\exp \left(t \Sigma^{0}(0)\right)=\exp (t \dot{\gamma}(0))=\dot{\gamma}(t)$. Moreover, if $V$ is the variation vector field of $\Sigma$, then

$$
\begin{aligned}
\left.\frac{d}{d s}\right|_{s=0} \sigma(s, t) & =\left.\frac{d}{d s}\right|_{s=0} \pi(\Sigma(s, t))=\left.T_{\dot{\gamma}(t)} \pi \cdot \frac{d}{d s}\right|_{s=0} \Sigma(s, t) \\
& =T_{\dot{\gamma}(t)} \pi \cdot V \in\left(\pi_{*} \mathcal{Q}\right)_{\gamma(t)} .
\end{aligned}
$$

That is, the variation vector field of $\sigma$ is precisely $T \pi \cdot V$ and it is a section of $\pi_{*} \mathcal{Q}$ along $\gamma$. Hence $\sigma$ is a $\left(\pi_{*} \mathcal{Q}\right)$ variation of $\gamma$. Conversely, let $\sigma$ be an $\mathcal{E}$-variation of $\gamma$ with variation vector field $J$. Let $\mathcal{Q}=\operatorname{span}\left\{X^{f}: X \in \Gamma(\mathcal{E})\right\}$; clearly, we have that $\mathcal{Q}$ is transversal to $\mathcal{V}$, that $\mathcal{H} \subseteq \mathcal{Q}$ (since $\mathcal{D} \subseteq \mathcal{E}$ ) and that $\pi_{*} \mathcal{Q}=\mathcal{E}$. Let

$$
\Sigma:(-\varepsilon, \varepsilon) \times[0, T] \rightarrow \mathcal{D}, \quad(s, t) \mapsto \Phi_{t}\left(\dot{\sigma}_{s}(0)\right) .
$$

Then $\Sigma_{s}$ is an integral curve of $\Xi$ for every $s \in(-\varepsilon, \varepsilon)$. Furthermore, we have $\Sigma_{0}(t)=\Phi_{t}\left(\dot{\sigma}_{0}(0)\right)=\Phi_{t}(\dot{\gamma}(0))=\dot{\gamma}(t)$. Let $V$ be the variation vector field of $\Sigma$; we have

$$
T_{\dot{\gamma}(t)} \pi \cdot V(t)=\left.\frac{d}{d s}\right|_{s=0} \pi\left(\Phi_{t}\left(\dot{\sigma}_{s}(0)\right)\right)=\left.\frac{d}{d s}\right|_{s=0} \exp _{\sigma_{s}(0)}\left(t \dot{\sigma}_{s}(0)\right)=J(t) \in \mathcal{E}_{\gamma(t)} .
$$

It follows that $V(t) \in(\mathcal{V} \oplus \mathcal{Q})_{\dot{\gamma}(t)}$, i.e., $\Sigma$ is a $\mathcal{Q}$-variation of $\dot{\gamma}$.

\section{Nonholonomic Jacobi fields}

Let $\left(\mathrm{M}, \mathcal{D}, \mathcal{D}^{\perp}, \mathbf{g}\right)$ be a nonholonomic Riemannian structure and let $\gamma:[0, T] \rightarrow \mathrm{M}$ be a nonholonomic geodesic. Let $\Sigma$ be a nonholonomic variation of $\dot{\gamma}$ and let $V$ denote the variation vector field of $\Sigma$. Let $\mathcal{I}$ be the subbundle of $\dot{\gamma}^{*}(\mathcal{V} \oplus \mathcal{H})$ given by

$$
\mathcal{I}_{t}=\left\{W_{t} \in(\mathcal{V} \oplus \mathcal{H})_{\dot{\gamma}(t)}: T_{\dot{\gamma}(t)} \Phi_{s} \cdot W_{t} \in(\mathcal{V} \oplus \mathcal{H})_{\dot{\gamma}(s+t)} \text { for every } s\right\}
$$

for $t \in[0, T]$. A nonholonomic Jacobi field along $\dot{\gamma}$ is a vector field $V \in \Gamma\left(\dot{\gamma}^{*}(\mathcal{V} \oplus \mathcal{H})\right)$ of the form

$$
V(t)=T_{\dot{\gamma}(0)} \Phi_{t} \cdot V_{0}, \quad V_{0} \in \mathcal{I}_{0} .
$$

Nonholonomic Jacobi fields are precisely the variation vector fields of nonholonomic variations of nonholonomic geodesics.

Proposition 3.1. If $V$ is the variation vector field of a nonholonomic variation of $\dot{\gamma}$, then it is a nonholonomic Jacobi field along $\dot{\gamma}$. Conversely, every nonholonomic Jacobi field along $\dot{\gamma}$ is the variation vector field of a nonholonomic variation of $\dot{\gamma}$. 
Proof. Let $V$ be the variation vector field of a nonholonomic variation $\Sigma$ of $\dot{\gamma}$. Since $\Sigma_{s}$ is an integral curve of $\Xi$ starting from $\Sigma_{s}(0)=\Sigma^{0}(s)$, we have $\Sigma(s, t)=\Phi_{t}\left(\Sigma^{0}(s)\right)$; hence

$$
V(t)=\left.\frac{d}{d s}\right|_{s=0} \Phi_{t}\left(\Sigma^{0}(s)\right)=T_{\dot{\gamma}(0)} \Phi_{t} \cdot \dot{\Sigma}^{0}(0),
$$

where $\dot{\Sigma}^{0}(0) \in(\mathcal{V} \oplus \mathcal{H})_{\dot{\gamma}(0)}$. That is, $V$ is a nonholonomic Jacobi field along $\dot{\gamma}$. For the converse, let $V$ be a nonholonomic Jacobi field along $\dot{\gamma}$; then there exists $V_{0} \in \mathcal{I}_{0}$ such that

$$
V(t)=T_{\dot{\gamma}(0)} \Phi_{t} \cdot V_{0}=\left.\frac{d}{d s}\right|_{s=0} \Phi_{t}(\lambda(s)),
$$

where $\lambda:(-\varepsilon, \varepsilon) \rightarrow \mathcal{D}$ is a curve such that $\lambda(0)=\dot{\gamma}(0)$ and $\dot{\lambda}(0)=V_{0}$. Define $\Sigma:(-\varepsilon, \varepsilon) \times[0, T] \rightarrow \mathcal{D}$ by $\Sigma(s, t)=$ $\Phi_{t}(\lambda(s))$. We have $\Sigma(0, t)=\Phi_{t}(\lambda(0))=\Phi_{t}(\dot{\gamma}(0))=\dot{\gamma}(t)$, and $\Sigma_{s}$ is clearly an integral curve of $\Xi$ for every $s \in(-\varepsilon, \varepsilon)$. Furthermore, by definition, $V$ is the variation vector field of $\Sigma$. Hence $\Sigma$ is a nonholonomic variation of $\dot{\gamma}$ (with variation vector field $V$ ).

Proposition 3.2. Let $V \in \Gamma\left(\dot{\gamma}^{*}(\mathcal{V} \oplus \mathcal{H})\right)$; the following statements are equivalent:

1. $V$ is a nonholonomic Jacobi field along $\dot{\gamma}$.

2. $V(s+t)=T_{\dot{\gamma}(t)} \Phi_{s} \cdot V(t)$ for every $s$ and $t$ for which both sides are defined.

3. $£_{\Xi} V=0$. (Here $£_{\Xi} V(t)=£_{\Xi} \widetilde{V}(\dot{\gamma}(t))$ for $t \in[0, T]$, where $\widetilde{V}$ is an extension of $V$ to an open neighbourhood of $\dot{\gamma}$.) Proof. (1.) $\Leftrightarrow$ (2.) Suppose $V$ is a nonholonomic Jacobi field along $\dot{\gamma}$, i.e., $V(t)=T_{\dot{\gamma}(0)} \Phi_{t} \cdot V_{0}$ for some $V_{0} \in \mathcal{I}_{0}$; since $\Phi_{s+t}=\Phi_{s} \circ \Phi_{t}$, we have

$$
V(s+t)=T_{\dot{\gamma}(0)} \Phi_{s+t} \cdot V_{0}=T_{\dot{\gamma}(t)} \Phi_{s} \cdot T_{\dot{\gamma}(0)} \Phi_{t} \cdot V_{0}=T_{\dot{\gamma}(t)} \Phi_{s} \cdot V(t) .
$$

(Moreover, it follows that $V \in \Gamma(\mathcal{I})$.) Conversely, if $V$ satisfies $V(s+t)=T_{\dot{\gamma}(t)} \Phi_{s} \cdot V(t)$, then (taking $t=0$ ) we have $V(s)=T_{\dot{\gamma}(0)} \Phi_{s} \cdot V(0)$. Since $V(s) \in(\mathcal{V} \oplus \mathcal{H})_{\dot{\gamma}(s)}$ for every $s \in[0, T]$, we have that $V(0) \in \mathcal{I}_{0}$. Thus $V$ is a nonholonomic Jacobi field.

(2.) $\Leftrightarrow$ (3.) Suppose that $V$ satisfies $V(s+t)=T_{\dot{\gamma}(t)} \Phi_{s} \cdot V(t)$; then

$$
\begin{aligned}
£_{\Xi V(t)} & =\left.\frac{d}{d s}\right|_{s=0} T_{\dot{\gamma}(s+t)} \Phi_{-s} \cdot V(s+t) \\
& =\left.\frac{d}{d s}\right|_{s=0} T_{\dot{\gamma}(s+t)} \Phi_{-s} \cdot T_{\dot{\gamma}(t)} \Phi_{s} \cdot V(t)=\left.\frac{d}{d s}\right|_{s=0} V(t)=0 .
\end{aligned}
$$

Conversely, suppose that $£_{\Xi} V=0$. Let $\lambda_{t}$ be the curve in $T_{\dot{\gamma}(t)} \mathcal{D}$ given by $\lambda_{t}(s)=T_{\dot{\gamma}(s+t)} \Phi_{-s} \cdot V(s+t)$; then $£_{\Xi} V=0$ if and only if $\dot{\lambda}_{t}(0)=0$ for every $t \in[0, T]$. We have

$$
\begin{aligned}
\dot{\lambda}_{t}\left(s_{0}\right) & =\left.\frac{d}{d s}\right|_{s=s_{0}} T_{\dot{\gamma}(s+t)} \Phi_{-s} \cdot V(s+t) \\
& =\left.\frac{d}{d \varepsilon}\right|_{\varepsilon=0} T_{\dot{\gamma}\left(\varepsilon+s_{0}+t\right)} \Phi_{-s_{0}-\varepsilon} \cdot V\left(\varepsilon+s_{0}+t\right) \\
& =\left.\frac{d}{d \varepsilon}\right|_{\varepsilon=0} T_{\dot{\gamma}\left(s_{0}+t\right)} \Phi_{-s_{0}} \cdot T_{\dot{\gamma}\left(\varepsilon+s_{0}+t\right)} \Phi_{-u} \cdot V\left(\varepsilon+s_{0}+t\right) \\
& =\left.T_{\dot{\gamma}\left(s_{0}+t\right)} \Phi_{-s_{0}} \cdot \frac{d}{d \varepsilon}\right|_{\varepsilon=0} T_{\dot{\gamma}\left(\varepsilon+s_{0}+t\right)} \Phi_{-\varepsilon} \cdot V\left(\varepsilon+s_{0}+t\right) \\
& =T_{\dot{\gamma}\left(s_{0}+t\right)} \Phi_{-s_{0}} \cdot £_{\Xi} V\left(s_{0}+t\right) \\
& =0 .
\end{aligned}
$$

That is, $\dot{\lambda}_{t}\left(s_{0}\right)=0$ for every $s_{0}$, hence $\lambda_{t}$ is constant; then

$$
T_{\dot{\gamma}(s+t)} \Phi_{-s} \cdot V(s+t)=\lambda_{t}(s)=\text { constant }=\lambda_{t}(0)=T_{\dot{\gamma}(t)} \Phi_{0} \cdot V(t)=V(t),
$$

whence $V(s+t)=T_{\dot{\gamma}(s+t)} \Phi_{s} \cdot V(t)$. 
Let $\left(E_{a}\right)$ be an orthonormal basis for $\mathcal{D}_{\gamma(0)}$ and let $\left(X_{a}\right)$ be the (orthonormal) frame for $\gamma^{*} \mathcal{D}$ obtained by parallel translating $\left(E_{a}\right)$ along $\gamma$, i.e., $X_{a}(t)=\Pi_{\gamma}^{t}\left(E_{a}\right)$. Lift $\left(X_{a}\right)$ to a frame $\left(X_{a}^{v}, X_{a}^{h}\right)$ for $\dot{\gamma}^{*}(\mathcal{V} \oplus \mathcal{H})$, where $X_{a}^{v} \in \Gamma\left(\dot{\gamma}^{*} \mathcal{V}\right)$ and $X_{a}^{h} \in \Gamma\left(\dot{\gamma}^{*} \mathcal{H}\right)$ are given by

$$
X_{a}^{v}(t)=v\left(\dot{\gamma}(t), X_{a}(t)\right) \quad \text { and } \quad X_{a}^{h}(t)=h\left(\dot{\gamma}(t), X_{a}(t)\right)
$$

for $t \in[0, T]$. Let $\widetilde{X}_{a} \in \Gamma(\mathcal{D})$ be an extension of $X_{a}$ to an open neighbourhood of $\gamma$; then $\widetilde{X}_{a}^{v}$ (resp. $\widetilde{X}_{a}^{h}$ ) is an extension of $X_{a}^{v}$ (resp. $X_{a}^{h}$ ) to an open neighbourhood of $\dot{\gamma}$.

Proposition 3.3. The frame $\left(X_{a}^{v}, X_{a}^{h}\right)$ satisfies the following equations:

$$
\left\{\begin{array}{l}
£_{\Xi} X_{a}^{v}=-X_{a}^{h} \\
£_{\Xi} X_{a}^{h}=v\left(\dot{\gamma}, K\left(X_{a} \wedge \dot{\gamma}\right) \dot{\gamma}\right)+f\left(\dot{\gamma}, £_{\dot{\gamma}}^{\mathscr{Q}} X_{a}\right) .
\end{array}\right.
$$

Proof. Let $\left(\nu^{a}\right)$ be the coframe dual to $\left(X_{a}\right)$, and let $\left(\eta^{a}\right)$ be the coframe dual to $\left(\widetilde{X}_{a}\right)$, so that $\eta_{\gamma(t)}^{a}=\nu_{t}^{a}$ for $t \in[0, T]$. We have $\Xi=\bar{\eta}^{a} \widetilde{X}_{a}^{h}$ (where $\bar{\eta}^{a} \in \mathcal{C}^{\infty}(\mathcal{D})$ is the function $\bar{\eta}^{a}\left(U_{q}\right)=\left\langle\eta_{q}^{a}, U_{q}\right\rangle$ ); indeed, if $U_{q}=u^{a} \widetilde{X}_{a}(q) \in \mathcal{D}$, then

$$
\Xi\left(U_{q}\right)=h\left(U_{q}, U_{q}\right)=h\left(U_{q}, u^{a} \widetilde{X}_{a}(q)\right)=u^{a} \widetilde{X}_{a}^{h}\left(U_{q}\right)=\bar{\eta}^{a}\left(U_{q}\right) \widetilde{X}_{a}^{h}\left(U_{q}\right) .
$$

For the first equation in (3.6), we get

$$
\begin{aligned}
£_{\Xi} X_{a}^{v} & =\left[\bar{\eta}^{b} \widetilde{X}_{b}^{h}, \widetilde{X}_{a}^{v}\right](\dot{\gamma})=\bar{\eta}^{b}(\dot{\gamma})\left[\widetilde{X}_{b}^{h}, \widetilde{X}_{a}^{v}\right](\dot{\gamma})-\widetilde{X}_{a}^{v}\left[\bar{\eta}^{b}\right](\dot{\gamma}) \widetilde{X}_{b}^{h}(\dot{\gamma}) \\
& =\bar{\nu}^{b}(\dot{\gamma})\left(\nabla_{\widetilde{X}_{b}} \widetilde{X}_{a}\right)^{v}(\dot{\gamma})-\left\langle\nu^{b}, X_{a}\right\rangle X_{b}^{h} \\
& =\dot{\gamma}^{b}\left(\nabla_{\widetilde{X}_{b}} \widetilde{X}_{a}\right)^{v}(\dot{\gamma})-X_{a}^{h} .
\end{aligned}
$$

Here $\dot{\gamma}^{b}$ are the components of $\dot{\gamma}$ with respect to $\left(X_{a}\right)$, i.e., $\dot{\gamma}=\dot{\gamma}^{b} X_{b}$. Thus

$$
£_{\Xi} X_{a}^{v}=v\left(\dot{\gamma}, \dot{\gamma}^{b} \nabla_{\widetilde{X}_{b}} \widetilde{X}_{a}(\dot{\gamma})\right)-X_{a}^{h}=v\left(\dot{\gamma}, \nabla_{\dot{\gamma}} X_{a}\right)-X_{a}^{h} .
$$

By definition, $X_{a}$ is parallel along $\gamma$; hence $\nabla_{\dot{\gamma}} X_{a}=0$, and so $£_{\Xi} X_{a}^{v}=-X_{a}^{h}$. For the second equation in (3.6), we get

$$
\begin{aligned}
£_{\Xi} X_{a}^{h}= & {\left[\bar{\eta}^{b} \widetilde{X}_{b}^{h}, \widetilde{X}_{a}^{h}\right](\dot{\gamma})=\dot{\gamma}^{b}\left[\widetilde{X}_{b}^{h}, \widetilde{X}_{a}^{h}\right](\dot{\gamma})-\widetilde{X}_{a}^{h}\left[\bar{\eta}^{b}\right](\dot{\gamma}) X_{b}^{h} } \\
= & \dot{\gamma}^{b}\left(\mathscr{V}\left(\left[\widetilde{X}_{b}^{h}, \widetilde{X}_{a}^{h}\right]\right)+(\mathscr{P}-\mathscr{V})\left(\left[\widetilde{X}_{b}^{h}, \widetilde{X}_{a}^{h}\right]\right)+\mathscr{Q}\left(\left[\widetilde{X}_{b}^{h}, \widetilde{X}_{a}^{h}\right]\right)\right)(\dot{\gamma}) \\
& \quad-\widetilde{X}_{a}^{h}\left[\bar{\eta}^{b}\right](\dot{\gamma}) X_{b}^{h} \\
= & \dot{\gamma}^{b}\left(\mathscr{V}\left(\left[\widetilde{X}_{b}^{h}, \widetilde{X}_{a}^{h}\right]\right)+\llbracket \widetilde{X}_{b}, \widetilde{X}_{a} \rrbracket^{h}+\mathscr{Q}\left(\left[\widetilde{X}_{b}, \widetilde{X}_{a}\right]\right)^{f}\right)(\dot{\gamma}) \\
& \quad-\widetilde{X}_{a}^{h}\left[\bar{\eta}^{b}\right](\dot{\gamma}) X_{b}^{h} \\
= & \dot{\gamma}^{b} \mathscr{V}\left(\left[\widetilde{X}_{b}^{h}, \widetilde{X}_{a}^{h}\right]\right)(\dot{\gamma})+h\left(\dot{\gamma}, \dot{\gamma}^{b} \llbracket \widetilde{X}_{b}, \widetilde{X}_{a} \rrbracket(\gamma)-\widetilde{X}_{a}^{h}\left[\bar{\eta}^{b}\right](\dot{\gamma}) X_{b}\right) \\
& \quad+f\left(\dot{\gamma}, \dot{\gamma}^{b} \mathscr{Q}\left(\left[\widetilde{X}_{b}, \widetilde{X}_{a}\right]\right)(\gamma)\right) .
\end{aligned}
$$

The horizontal term vanishes; indeed, we have

$$
\begin{aligned}
\dot{\gamma}^{b} \llbracket \widetilde{X}_{b}, \widetilde{X}_{a} \rrbracket(\gamma) & =\dot{\gamma}^{b} \nabla_{\widetilde{X}_{b}} \widetilde{X}_{a}(\gamma)-\dot{\gamma}^{b} \nabla_{\widetilde{X}_{a}} \widetilde{X}_{b}(\gamma) \\
& =\nabla_{\dot{\gamma}} X_{a}-\dot{\gamma}^{b} \nabla_{\widetilde{X}_{a}} \widetilde{X}_{b}(\gamma) \\
& =-\dot{\gamma}^{b} \nabla_{\widetilde{X}_{a}} \widetilde{X}_{b}(\gamma) .
\end{aligned}
$$

Likewise, if $Z=z^{b} \widetilde{X}_{b} \in \Gamma(\mathcal{D})$ is an extension of $\dot{\gamma}$ to an open neighbourhood of $\gamma$, where $z^{b} \in \mathcal{C}^{\infty}(\mathrm{M})$, then

$$
\begin{aligned}
\widetilde{X}_{a}^{h}\left[\bar{\eta}^{b}\right](\dot{\gamma}) X_{b} & =\left\langle\nabla_{\widetilde{X}_{a}} \eta^{b}, Z\right\rangle(\gamma) X_{b}=\widetilde{X}_{a}\left[\left\langle\eta^{b}, Z\right\rangle\right](\gamma) X_{b}-\left\langle\eta^{b}, \nabla_{\widetilde{X}_{a}} Z\right\rangle(\gamma) X_{b} \\
& =\widetilde{X}_{a}\left[z^{b}\right](\gamma) X_{b}-\left\langle\eta^{b}, \widetilde{X}_{a}\left[z^{c}\right] \widetilde{X}_{c}+z^{c} \nabla_{\widetilde{X}_{a}} \widetilde{X}_{c}\right\rangle(\gamma) X_{b} \\
& =\widetilde{X}_{a}\left[z^{b}\right](\gamma) X_{b}-\widetilde{X}_{a}\left[z^{c}\right](\gamma)\left\langle\eta^{b}, \widetilde{X}_{c}\right\rangle(\gamma) X_{b}-\left\langle\eta^{b}, z^{c} \nabla_{\widetilde{X}_{a}} \widetilde{X}_{c}\right\rangle(\gamma) X_{b} \\
& =-\dot{\gamma}^{c}\left\langle\eta^{b}, \nabla_{\widetilde{X}_{a}} \widetilde{X}_{c}\right\rangle(\gamma) X_{b} \\
& =-\dot{\gamma}^{c} \nabla_{\widetilde{X}_{a}} \widetilde{X}_{c}(\gamma) .
\end{aligned}
$$


Hence $\dot{\gamma}^{b} \llbracket \widetilde{X}_{b}, \widetilde{X}_{a} \rrbracket(\gamma)-\widetilde{X}_{a}^{h}\left[\bar{\eta}^{b}\right](\dot{\gamma}) X_{b}=0$, i.e., the horizontal term in $£_{\Xi} X_{a}^{h}$ vanishes. Thus

$$
\begin{aligned}
£_{\Xi} X_{a}^{h} & =\dot{\gamma}^{b} \mathscr{V}\left(\left[\widetilde{X}_{b}^{h}, \widetilde{X}_{a}^{h}\right]\right)(\dot{\gamma})+f\left(\dot{\gamma}, \dot{\gamma}^{b} \mathscr{Q}\left(\left[\widetilde{X}_{b}, \widetilde{X}_{a}\right]\right)(\gamma)\right) \\
& =-\dot{\gamma}^{b} v\left(\dot{\gamma}, K\left(\widetilde{X}_{b} \wedge \widetilde{X}_{a}\right) \dot{\gamma}\right)+f\left(\dot{\gamma}, £_{\dot{\gamma}}^{\mathcal{Q}} X_{a}\right) \\
& =v\left(\dot{\gamma}, K\left(X_{a} \wedge \dot{\gamma}\right) \dot{\gamma}\right)+f\left(\dot{\gamma}, £_{\dot{\gamma}}^{\mathcal{Q}} X_{a}\right) .
\end{aligned}
$$

We are now in a position to derive the defining equations for a nonholonomic variation vector field along (the base curve) $\gamma$.

Theorem 3.1. If $V$ is a nonholonomic Jacobi field along $\dot{\gamma}$, then the nonholonomic variation vector field (along $\gamma$ ) $J=T \pi \cdot V \in \Gamma\left(\gamma^{*} \mathcal{D}\right)$ satisfies

$$
\nabla_{\dot{\gamma}} \nabla_{\dot{\gamma}} J+\Psi_{\dot{\gamma}}(J)=0 \quad \text { and } £_{\dot{\gamma}}^{\mathcal{Q}} J=0 .
$$

Conversely, if $J \in \Gamma\left(\gamma^{*} \mathcal{D}\right)$ satisfies (3.9), then there exists a unique nonholonomic Jacobi field $V$ along $\dot{\gamma}$ such that $J=T \pi \cdot V$.

Proof. Let $V=x^{a} X_{a}^{v}+y^{a} X_{a}^{h} \in \Gamma\left(\dot{\gamma}^{*}(\mathcal{V} \oplus \mathcal{H})\right)$ for $x^{a}, y^{a} \in \mathcal{C}^{\infty}([0, T])$, and let $J=T \pi \cdot V=y^{a} X_{a}$. Let $\widetilde{V}=\widetilde{x}^{a} \widetilde{X}_{a}^{v}+$ $\widetilde{y}^{a} \widetilde{X}_{a}^{h}$ be an extension of $V$ to an open neighbourhood of $\dot{\gamma}$. We have

$$
\begin{aligned}
£_{\Xi V}= & {[\Xi, \widetilde{V}](\dot{\gamma})=\left[\Xi, \widetilde{x}^{a} \widetilde{X}_{a}^{v}+\widetilde{y}^{a} \widetilde{X}_{a}^{h}\right](\dot{\gamma}) } \\
= & \left.\Xi \widetilde{x}^{a}\right](\dot{\gamma}) X_{a}^{v}+\Xi\left[\widetilde{y}^{a}\right](\dot{\gamma}) X_{a}^{h}+x^{a} £_{\Xi} X_{a}^{v}+y^{a} £_{\Xi} X_{a}^{h} \\
= & \Xi\left[\widetilde{x}^{a}\right](\dot{\gamma}) X_{a}^{v}+\Xi\left[\widetilde{y}^{a}\right](\dot{\gamma}) X_{a}^{h}-x^{a} X_{a}^{h}+y^{a} v\left(\dot{\gamma}, K\left(X_{a} \wedge \dot{\gamma}\right) \dot{\gamma}\right) \\
& \quad+y^{a} f\left(\dot{\gamma}, £_{\dot{\gamma}}^{\mathcal{Q}} X_{a}\right) \\
= & v\left(\dot{\gamma}, \Xi\left[\widetilde{x}^{a}\right](\dot{\gamma}) X_{a}+K(J \wedge \dot{\gamma}) \dot{\gamma}\right)+h\left(\dot{\gamma}, \Xi\left[\widetilde{y}^{a}\right](\dot{\gamma}) X_{a}-x^{a} X_{a}\right)+f\left(\dot{\gamma}, £_{\dot{\gamma}}^{\mathscr{Q}} J\right) .
\end{aligned}
$$

Since $\dot{\gamma}$ is an integral curve of $\Xi$, we get $\Xi\left[\widetilde{x}^{a}\right](\dot{\gamma}(t))=\left.\frac{d}{d s}\right|_{s=0} \widetilde{x}^{a}(\dot{\gamma}(s+t))=\dot{x}^{a}(t)$ and $\Xi\left[\widetilde{y}^{a}\right](\dot{\gamma}(t))=\dot{y}^{a}(t)$; hence

$$
£_{\Xi} V=v\left(\dot{\gamma}, \dot{x}^{a} X_{a}+K(J \wedge \dot{\gamma}) \dot{\gamma}\right)+h\left(\dot{\gamma},\left(\dot{y}^{a}-x^{a}\right) X_{a}\right)+f\left(\dot{\gamma}, £_{\dot{\gamma}}^{\mathscr{Q}} J\right) .
$$

Suppose that $V$ is a nonholonomic Jacobi field along $\dot{\gamma}$, i.e., $£ \Xi V=0$; then $\dot{x}^{a} X_{a}+K(J \wedge \dot{\gamma}) \dot{\gamma}=0, x^{a}=\dot{y}^{a}$ and $£_{\dot{\gamma}}^{\mathscr{Q}} J=0$. Using the fact that $X_{a}$ is parallel along $\gamma$, we get

$$
\nabla_{\dot{\gamma}} \nabla_{\dot{\gamma}} J=\nabla_{\dot{\gamma}} \nabla_{\dot{\gamma}}\left(y^{a} X_{a}\right)=\nabla_{\dot{\gamma}}\left(\dot{y}^{a} X_{a}\right)=\ddot{y}^{a} X_{a}=\dot{x}^{a} X_{a}=-K(J \wedge \dot{\gamma}) \dot{\gamma} .
$$

We claim that $C(J \wedge \dot{\gamma}) \dot{\gamma}=0$. Indeed, let $Z, \widetilde{J} \in \Gamma(\mathcal{D})$ be extensions of $\dot{\gamma}$ and $J$, respectively, to an open neighbourhood of $\gamma$, and let $L \in \Gamma\left(\gamma^{*} \mathcal{D}\right)$ be arbitrary. Since $£_{\dot{\gamma}}^{\mathscr{Q}} J=\mathscr{Q}\left(£_{\dot{\gamma}} J\right)=0$, it follows that $\mathscr{Q}([\widetilde{J}, Z])$ vanishes along $\gamma$. Moreover, we have that $£^{\mathscr{P}}: \Gamma\left(\mathcal{D}^{\perp}\right) \times \Gamma(\mathcal{D}) \rightarrow \Gamma(\mathcal{D})$ is a $\mathcal{D}^{\perp}$-connection on $\mathcal{D}$; consequently,

$$
\widehat{C}(J, \dot{\gamma}, \dot{\gamma}, L)=\frac{1}{2}\left(£_{\mathscr{Q}([\widetilde{J}, Z])(\gamma)}^{\mathscr{P}} \mathbf{g}\right)(\dot{\gamma}, L)=0,
$$

whence $C(J \wedge \dot{\gamma}) \dot{\gamma}=0$. Therefore, if $V$ is a Jacobi field along $\dot{\gamma}$, then $J=T \pi \cdot V$ satisfies (3.9).

Conversely, suppose that $J=y^{a} X_{a} \in \Gamma\left(\gamma^{*} \mathcal{D}\right)$ satisfies (3.9); then one may verify that the vector field $V=\dot{y}^{a} X_{a}^{v}+y^{a} X_{a}^{h} \in \Gamma\left(\dot{\gamma}^{*}(\mathcal{V} \oplus \mathcal{H})\right)$ satisfies $£ \Xi V=0$, i.e., $V$ is a nonholonomic Jacobi field along $\dot{\gamma}$. It remains to prove uniqueness. Let $\bar{V}=\bar{x}^{a} X_{a}^{v}+\bar{y}^{a} X_{a}^{h}$ be another nonholonomic Jacobi field along $\dot{\gamma}$ such that $T \pi \cdot \bar{V}=J$. Since $£_{\Xi} \bar{V}=0$, we get $\bar{x}^{a}=\dot{y}^{a}$; furthermore, as $\bar{y}^{a} X_{a}=T \pi \cdot \bar{V}=J=y^{a} X_{a}$, we have $\bar{y}^{a}=y^{a}$, whence $\bar{x}^{a}=x^{a}$. Thus $\bar{V}=V$, and so $V$ is unique.

Hence, in accordance with Theorem 3.1, we make the following definition. A vector field $J \in \Gamma\left(\gamma^{*} \mathcal{D}\right)$ is called a nonholonomic Jacobi field along $\gamma$ if

$$
\nabla_{\dot{\gamma}} \nabla_{\dot{\gamma}} J+\Psi_{\dot{\gamma}}(J)=0 \quad \text { and } \quad £_{\dot{\gamma}}^{\mathcal{Q}} J=0 .
$$

It should be clear that there exists a one-to-one correspondence between nonholonomic Jacobi fields along $\dot{\gamma}$ and nonholonomic Jacobi fields along $\gamma$. Since the Jacobi operators completely determine the component $R$ of the Schouten curvature tensor, it follows that $R$ is completely determined by the nonholonomic Jacobi field equation (3.12).

Remark 3.1. In [6] a geometric interpretation was obtained for the component $C$ of the Schouten curvature tensor. (It turns out that $C$ measures the "geodesic invariance" of the nonholonomic Riemannian structure.) Theorem 3.1 gives a geometric interpretation for the remaining component $R$ : it controls the evolution of one-parameter families (specifically, nonholonomic variations) of nonholonomic geodesics. 


\section{Restricted Jacobi fields}

Let $\left(\mathrm{M}, \mathcal{D}, \mathcal{D}^{\perp}, \mathrm{g}\right)$ be a Wagner structure, where $\mathcal{D}$ has degree of nonholonomy $N$. Fix $1 \leq i \leq N-1$, let $\gamma:[0, T] \rightarrow$ M be a nonholonomic geodesic, and let $\Sigma:(-\varepsilon, \varepsilon) \times[0, T] \rightarrow \mathcal{D}$ be an $\mathcal{H}^{i+1}$-variation of $\dot{\gamma}$. Let $\mathcal{I}^{i+1}$ be the subbundle of $\dot{\gamma}^{*}\left(\mathcal{V} \oplus \mathcal{H}^{i+1}\right)$ given by

$$
\mathcal{I}_{t}^{i+1}=\left\{V_{t} \in\left(\mathcal{V} \oplus \mathcal{H}^{i}\right)_{\dot{\gamma}(t)}: T_{\dot{\gamma}(t)} \Phi_{s} \cdot V_{t} \in\left(\mathcal{V} \oplus \mathcal{H}^{i}\right)_{\dot{\gamma}(s+t)} \text { for every } s\right\}
$$

for $t \in[0, T]$. An $\mathcal{H}^{i+1}$-restricted Jacobi field along $\dot{\gamma}$ (or simply an $\mathcal{H}^{i+1}$-Jacobi field along $\dot{\gamma}$ ) is a vector field $V \in \Gamma\left(\dot{\gamma}^{*}\left(\mathcal{V} \oplus \mathcal{H}^{i+1}\right)\right)$ of the form

$$
V(t)=T_{\dot{\gamma}(0)} \Phi_{t} \cdot V_{0}, \quad V_{0} \in \mathcal{I}_{0}^{i+1} .
$$

We also define an $\mathcal{H}^{1}$-Jacobi field along $\dot{\gamma}$ to be a nonholonomic Jacobi field along $\dot{\gamma} \cdot \mathcal{H}^{i+1}$-Jacobi fields are precisely the variation vector fields of $\mathcal{H}^{i+1}$-variations of nonholonomic geodesics. (The proofs of the following two results are very similar to those of Proposition 3.1 and Proposition 3.2, respectively, and hence are omitted.)

Proposition 4.1. If $V$ is the variation vector field of an $\mathcal{H}^{i+1}$-variation of $\dot{\gamma}$, then it is an $\mathcal{H}^{i+1}$-Jacobi field along $\dot{\gamma}$. Conversely, every $\mathcal{H}^{i+1}$-Jacobi field along $\dot{\gamma}$ is the variation vector field of an $\mathcal{H}^{i+1}$-variation of $\dot{\gamma}$.

Proposition 4.2. Let $V \in \Gamma\left(\dot{\gamma}^{*}\left(\mathcal{V} \oplus \mathcal{H}^{i+1}\right)\right)$; the following statements are equivalent:

1. $V$ is an $\mathcal{H}^{i}$-Jacobi field along $\dot{\gamma}$.

2. $V(s+t)=T_{\dot{\gamma}(t)} \Phi_{s} \cdot V(t)$ for every s and $t$ for which both sides are defined.

3. $£_{\Xi} V=0$.

Fix $1 \leq i \leq N-1$ and let the index $j$ run through $1, \ldots, i$. Let $\left(E_{a_{0}}^{0}\right)$ be an orthonormal basis for $\mathcal{D}_{\gamma(0)}$ and let $\left(E_{a_{j}}^{j}\right)$ be a basis for $\mathcal{E}_{\gamma(0)}^{j}$. In order to define a moving frame for $\gamma^{*} \mathcal{D}^{i+1}$, we require a means of parallel translating the basis $\left(E_{a_{j}}^{j}\right)$ along $\gamma$. To that end, notice that the mapping

$$
\Gamma(\mathcal{D}) \times \Gamma\left(\mathcal{E}^{j}\right) \rightarrow \Gamma\left(\mathcal{E}^{j}\right), \quad(U, X) \mapsto £_{U}^{\mathscr{Q}_{j}} X
$$

is a restricted connection; let $P_{\gamma}^{j, t}: \mathcal{E}_{\gamma(0)}^{j} \rightarrow \mathcal{E}_{\gamma(t)}^{j}$ be its associated parallel transport. Hence, let $\left(X_{a_{0}}^{0}\right)$ and $\left(X_{a_{j}}^{j}\right)$ be the frames along $\gamma$ obtained by parallel translating $\left(E_{a_{0}}^{0}\right)$ and $\left(E_{a_{j}}^{j}\right)$ along $\gamma$, respectively, i.e.,

$$
X_{a_{0}}^{0}(t)=\Pi_{\gamma}^{t}\left(E_{a_{0}}^{0}\right) \quad \text { and } \quad X_{a_{j}}^{j}(t)=P_{\gamma}^{j, t}\left(E_{a_{j}}^{j}\right) .
$$

Lift $\left(X_{a_{0}}^{0}, X_{a_{1}}^{1}, \ldots, X_{a_{i}}^{i}\right)$ to a frame $\left(\left(X_{a_{0}}^{0}\right)^{v},\left(X_{a_{0}}^{0}\right)^{h},\left(X_{a_{1}}^{1}\right)^{h^{2}}, \ldots,\left(X_{a_{i}}^{i}\right)^{h^{i+1}}\right)$ for $\dot{\gamma}^{*}\left(\mathcal{V} \oplus \mathcal{H}^{i+1}\right)$, where $\left(X_{a_{0}}^{0}\right)^{v} \in$ $\Gamma\left(\dot{\gamma}^{*} \mathcal{V}\right),\left(X_{a_{0}}^{0}\right)^{h} \in \Gamma\left(\dot{\gamma}^{*} \mathcal{H}\right)$ and $\left(X_{a_{j}}^{j}\right)^{h^{j+1}} \in \Gamma\left(\dot{\gamma}^{*} \mathcal{Q}^{j}\right)$ are given by

$$
\begin{gathered}
\left(X_{a_{0}}^{0}\right)^{v}(t)=v\left(\dot{\gamma}(t), X_{a_{0}}^{0}(t)\right), \quad\left(X_{a_{0}}^{0}\right)^{h}(t)=h\left(\dot{\gamma}(t), X_{a_{0}}^{0}(t)\right), \\
\text { and }\left(X_{a_{j}}^{j}\right)^{h^{j+1}}(t)=h^{j+1}\left(\dot{\gamma}(t), X_{a_{j}}^{j}(t)\right)
\end{gathered}
$$

for $t \in[0, T]$. Let $\widetilde{X}_{a_{0}}^{0} \in \Gamma(\mathcal{D})$ (resp. $\widetilde{X}_{a_{j}}^{j} \in \Gamma\left(\mathcal{E}^{j}\right)$ ) be an extension of $X_{a_{0}}^{0}$ (resp. $X_{a_{j}}^{j}$ ) to an open neighbourhood of $\gamma$; then $\left(\widetilde{X}_{a_{0}}^{0}\right)^{v}$ (resp. $\left(\widetilde{X}_{a_{0}}^{0}\right)^{h}$, resp. $\left.\left(\widetilde{X}_{a_{j}}^{j}\right)^{j+1}\right)$ is an extension of $\left(X_{a_{0}}^{0}\right)^{v}\left(\operatorname{resp} .\left(X_{a_{0}}^{0}\right)^{h}\right.$, resp. $\left.\left(X_{a_{j}}^{j}\right)^{h^{j+1}}\right)$ to an open neighbourhood of $\dot{\gamma}$.

Proposition 4.3. The frame $\left(\left(X_{a_{0}}^{0}\right)^{v},\left(X_{a_{0}}^{0}\right)^{h^{1}},\left(X_{a_{1}}^{1}\right)^{h^{2}}, \ldots,\left(X_{a_{i}}^{i}\right)^{h^{i+1}}\right)$ satisfies the following equations. For $\left(X_{a_{0}}^{0}\right)^{v}$ and $\left(X_{a_{0}}^{0}\right)^{h^{1}}$, we have:

$$
\left\{\begin{aligned}
£_{\Xi}\left(X_{a_{0}}^{0}\right)^{v} & =-\left(X_{a_{0}}^{0}\right)^{h^{1}} \\
£ \Xi\left(X_{a_{0}}^{0}\right)^{h^{1}} & =v\left(\dot{\gamma}, K^{1}\left(X_{a_{0}}^{0} \wedge \dot{\gamma}\right) \dot{\gamma}\right)+h^{2}\left(\dot{\gamma}, £_{\dot{\gamma}}^{\mathscr{Q}_{1}} X_{a_{0}}^{0}\right) .
\end{aligned}\right.
$$

The corresponding equations for the remaining elements $\left(X_{a_{1}}^{1}\right)^{h^{2}}, \ldots,\left(X_{a_{i}}^{i}\right)^{h^{i+1}}$ are given as follows:

1. If $N=2$, then

$$
£_{\Xi}\left(X_{a_{1}}^{1}\right)^{h^{2}}=v\left(\dot{\gamma}, K^{2}\left(X_{a_{1}}^{1} \wedge \dot{\gamma}\right) \dot{\gamma}\right)+h^{1}\left(\dot{\gamma}, K^{1}\left(\Theta_{1}\left(X_{a_{1}}^{1}\right)\right) \dot{\gamma}\right)
$$


2. If $N>2$ and $i=1$, then

$$
\begin{aligned}
£_{\Xi}\left(X_{a_{1}}^{1}\right)^{h^{2}=v} & \left(\dot{\gamma}, K^{2}\left(X_{a_{1}}^{1} \wedge \dot{\gamma}\right) \dot{\gamma}\right)+h^{1}\left(\dot{\gamma}, K^{1}\left(\Theta_{1}\left(X_{a_{1}}^{1}\right)\right) \dot{\gamma}\right) \\
& +h^{3}\left(\dot{\gamma}, £_{\dot{\gamma}}^{\mathscr{Q}_{2}} X_{a_{1}}^{1}\right) .
\end{aligned}
$$

3. If $N>2$ and $1<i<N-1$, then

$$
\left\{\begin{aligned}
£_{\Xi}\left(X_{a_{1}}^{1}\right)^{h^{2}=v} & \left(\dot{\gamma}, K^{2}\left(X_{a_{1}}^{1} \wedge \dot{\gamma}\right) \dot{\gamma}\right)+h^{1}\left(\dot{\gamma}, K^{1}\left(\Theta_{1}\left(X_{a_{1}}^{1}\right)\right) \dot{\gamma}\right) \\
& +h^{3}\left(\dot{\gamma}, £_{\dot{\gamma}}^{\mathscr{Q}_{2}} X_{a_{1}}^{1}\right) \\
£_{\Xi}\left(X_{a_{j}}^{j}\right)^{h^{j+1}}=v & \left(\dot{\gamma}, K^{j+1}\left(X_{a_{j}}^{j} \wedge \dot{\gamma}\right) \dot{\gamma}\right)+h^{1}\left(\dot{\gamma}, K^{j}\left(\Theta_{j}\left(X_{a_{j}}^{j}\right)\right) \dot{\gamma}\right) \\
& +\sum_{k=1}^{j-1} h^{k+1}\left(\dot{\gamma}, £_{\dot{\gamma}}^{\mathcal{Q}_{k}} X_{a_{j}}^{j}\right)+h^{j+2}\left(\dot{\gamma}, £_{\dot{\gamma}}^{\mathcal{Q}_{j+1}} X_{a_{j}}^{j}\right)
\end{aligned}\right.
$$

for $j=2, \ldots, i$.

4. If $N>2$ and $i=N-1$, then

$$
\left\{\begin{aligned}
£_{\Xi}\left(X_{a_{1}}^{1}\right)^{h^{2}=v} & \left(\dot{\gamma}, K^{2}\left(X_{a_{1}}^{1} \wedge \dot{\gamma}\right) \dot{\gamma}\right)+h^{1}\left(\dot{\gamma}, K^{1}\left(\Theta_{1}\left(X_{a_{1}}^{1}\right)\right) \dot{\gamma}\right) \\
& +h^{3}\left(\dot{\gamma}, £_{\dot{\gamma}}^{\mathscr{Q}_{2}} X_{a_{1}}^{1}\right) \\
£_{\Xi}\left(X_{a_{j}}^{j}\right)^{h^{j+1}}=v( & \left(\dot{\gamma}, K^{j+1}\left(X_{a_{j}}^{j} \wedge \dot{\gamma}\right) \dot{\gamma}\right)+h^{1}\left(\dot{\gamma}, K^{j}\left(\Theta_{j}\left(X_{a_{j}}^{j}\right)\right) \dot{\gamma}\right) \\
& +\sum_{k=1}^{j-1} h^{k+1}\left(\dot{\gamma}, £_{\dot{\gamma}}^{\mathcal{Q}_{k}} X_{a_{j}}^{j}\right)+h^{j+2}\left(\dot{\gamma}, £_{\dot{\gamma}}^{\mathscr{Q}_{j+1}} X_{a_{j}}^{j}\right) \\
£_{\Xi}\left(X_{a_{N-1}}^{N-1}\right)^{h^{N}=v} & \left(\dot{\gamma}, K^{N}\left(X_{a_{N-1}}^{N-1} \wedge \dot{\gamma}\right) \dot{\gamma}\right) \\
& +h^{1}\left(\dot{\gamma}, K^{N-1}\left(\Theta_{N-1}\left(X_{a_{N-1}}^{N-1}\right)\right) \dot{\gamma}\right) \\
& +\sum_{k=1}^{N-2} h^{k+1}\left(\dot{\gamma}, £_{\dot{\gamma}}^{\mathscr{Q}_{k}} X_{a_{N-1}}^{N-1}\right)
\end{aligned}\right.
$$

for $j=2, \ldots, N-2$.

Proof. Let $\left(\nu_{0}^{a_{0}}\right)$ be the coframe dual to $\left(X_{a_{0}}^{0}\right)$ and let $\left(\eta_{0}^{a_{0}}\right)$ be the coframe dual to $\left(\widetilde{X}_{a_{0}}^{0}\right)$, so that $\left(\eta_{0}^{a_{0}}\right)_{\gamma(t)}=\left(\nu_{0}^{a_{0}}\right)_{t}$ for $t \in[0, T]$. A similar argument to that in the proof of Proposition 3.3 yields the first equation in (4.5). For the second equation, we have $\Xi=\bar{\eta}_{0}^{a_{0}}\left(\widetilde{X}_{a_{0}}^{0}\right)^{h}$; hence

$$
\begin{aligned}
£ \equiv\left(X_{a_{0}}^{0}\right)^{h^{1}} & =\left[\bar{\eta}_{0}^{b_{0}}\left(\widetilde{X}_{b_{0}}^{0}\right)^{h^{1}},\left(\widetilde{X}_{a_{0}}^{0}\right)^{h^{1}}\right](\dot{\gamma}) \\
& =\dot{\gamma}^{b_{0}}\left[\left(\widetilde{X}_{b_{0}}^{0}\right)^{h^{1}},\left(\widetilde{X}_{a_{0}}^{0}\right)^{h^{1}}\right](\dot{\gamma})-\left(\widetilde{X}_{a_{0}}^{0}\right)^{h^{1}}\left[\bar{\eta}_{0}^{b_{0}}\right](\dot{\gamma})\left(X_{b_{0}}^{0}\right)^{h^{1}} .
\end{aligned}
$$

(Here $\dot{\gamma}^{b_{0}}$ are the components of $\dot{\gamma}$ with respect to $\left(X_{a_{0}}^{0}\right)$.) Since $[\mathcal{D}, \mathcal{D}] \subsetneq \mathcal{D}^{2}$, it follows that $\left[\widetilde{X}_{b_{0}}^{0}, \widetilde{X}_{a_{0}}^{0}\right] \in \Gamma\left(\mathcal{D}^{2}\right)$, and hence $\left[\left(\widetilde{X}_{b_{0}}^{0}\right)^{h^{1}},\left(\widetilde{X}_{a_{0}}^{0}\right)^{h^{1}}\right] \in \Gamma\left(\mathcal{V} \oplus \mathcal{H}^{2}\right)$. Thus

$$
\begin{aligned}
£_{\Xi}\left(X_{a_{0}}^{0}\right)^{h^{1}}= & \dot{\gamma}^{b_{0}}\left(\mathscr{V}\left(\left[\left(\widetilde{X}_{b_{0}}^{0}\right)^{h^{1}},\left(\widetilde{X}_{a_{0}}^{0}\right)^{h^{1}}\right]\right)+(\mathscr{P}-\mathscr{V})\left(\left[\left(\widetilde{X}_{b_{0}}^{0}\right)^{h^{1}},\left(\widetilde{X}_{a_{0}}^{0}\right)^{h^{1}}\right]\right)\right. \\
& \left.+\mathscr{Q}_{1}\left(\left[\left(\widetilde{X}_{b_{0}}^{0}\right)^{h^{1}},\left(\widetilde{X}_{a_{0}}^{0}\right)^{h^{1}}\right]\right)\right)(\dot{\gamma})-\left(\widetilde{X}_{a_{0}}^{0}\right)^{h^{1}}\left[\bar{\eta}_{0}^{b_{0}}\right](\dot{\gamma})\left(X_{b_{0}}^{0}\right)^{h^{1}} \\
= & \dot{\gamma}^{b_{0}} \mathscr{V}\left(\left[\left(\widetilde{X}_{b_{0}}^{0}\right)^{h^{1}},\left(\widetilde{X}_{a_{0}}^{0}\right)^{h^{1}}\right]\right)(\dot{\gamma})+\dot{\gamma}^{b_{0}} \llbracket \widetilde{X}_{b_{0}}^{0}, \widetilde{X}_{a_{0}}^{0} \rrbracket^{h^{1}}(\dot{\gamma}) \\
& +\dot{\gamma}^{b_{0}} \mathscr{Q}_{1}\left(\left[\widetilde{X}_{b_{0}}^{0}, \widetilde{X}_{a_{0}}^{0}\right]\right)^{h^{2}}(\dot{\gamma})-\left(\widetilde{X}_{a_{0}}^{0}\right)^{h^{1}}\left[\bar{\eta}_{0}^{b_{0}}\right](\dot{\gamma})\left(X_{b_{0}}^{0}\right)^{h^{1}} \\
= & \dot{\gamma}^{b_{0}} \mathscr{V}\left(\left[\left(\widetilde{X}_{b_{0}}^{0}\right)^{h^{1}},\left(\widetilde{X}_{a_{0}}^{0}\right)^{h^{1}}\right]\right)(\dot{\gamma}) \\
& +h^{1}\left(\dot{\gamma}, \dot{\gamma}^{b_{0}} \llbracket \widetilde{X}_{b_{0}}^{0}, \widetilde{X}_{a_{0}}^{0} \rrbracket(\gamma)-\left(\widetilde{X}_{a_{0}}^{0}\right)^{h^{1}}\left[\bar{\eta}_{0}^{b_{0}}\right](\dot{\gamma}) X_{b_{0}}^{0}\right) \\
& +h^{2}\left(\dot{\gamma}, \dot{\gamma}^{b_{0}} \mathscr{Q}_{1}\left(\left[\widetilde{X}_{b_{0}}^{0}, \widetilde{X}_{a_{0}}^{0}\right]\right)(\gamma)\right) .
\end{aligned}
$$


As in the proof of Proposition 3.3, one can show that the $h^{1}$-term vanishes, i.e., $\dot{\gamma}^{b_{0}} \llbracket \widetilde{X}_{b_{0}}^{0}, \widetilde{X}_{a_{0}}^{0} \rrbracket(\gamma)=$

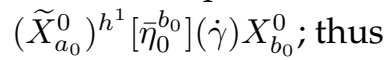

$$
\begin{aligned}
£_{\Xi}\left(X_{a_{0}}^{0}\right)^{h^{1}} & =\dot{\gamma}^{b_{0}} \mathscr{V}\left(\left[\left(\widetilde{X}_{b_{0}}^{0}\right)^{h^{1}},\left(\widetilde{X}_{a_{0}}^{0}\right)^{h^{1}}\right]\right)(\dot{\gamma})+h^{2}\left(\dot{\gamma}, \dot{\gamma}^{b_{0}} \mathscr{Q}_{1}\left(\left[\widetilde{X}_{b_{0}}^{0}, \widetilde{X}_{a_{0}}^{0}\right]\right)(\gamma)\right) \\
& =-\dot{\gamma}^{b_{0}} v\left(\dot{\gamma}, K\left(\widetilde{X}_{b_{0}}^{0} \wedge \widetilde{X}_{a_{0}}^{0}\right) \dot{\gamma}\right)+h^{2}\left(\dot{\gamma}, £_{\dot{\gamma}}^{\mathscr{Q}_{1}} X_{a_{0}}^{0}\right) \\
& =v\left(\dot{\gamma}, K\left(X_{a_{0}}^{0} \wedge \dot{\gamma}\right) \dot{\gamma}\right)+h^{2}\left(\dot{\gamma}, £_{\dot{\gamma}}^{\mathscr{Q}_{1}} X_{a_{0}}^{0}\right),
\end{aligned}
$$

i.e., we have the second equation in (4.5). It remains to establish the equations (4.6), (4.7), (4.8), and (4.9). For brevity, we shall prove only (4.9); the other cases are proved similarly. Suppose that $N>2$ and $i=N-1$, and let $1 \leq j \leq N-1$; then

$$
\begin{aligned}
£_{\Xi}\left(X_{a_{j}}^{j}\right)^{h^{j+1}} & =\left[\bar{\eta}_{0}^{b_{0}}\left(\widetilde{X}_{b_{0}}^{0}\right)^{h^{1}},\left(\widetilde{X}_{a_{j}}^{j}\right)^{h^{j+1}}\right](\dot{\gamma}) \\
& =\dot{\gamma}^{b_{0}}\left[\left(\widetilde{X}_{b_{0}}^{0}\right)^{h^{1}},\left(\widetilde{X}_{a_{j}}^{j}\right)^{h^{j+1}}\right](\dot{\gamma})-\left(\widetilde{X}_{a_{j}}^{j}\right)^{h^{j+1}}\left[\bar{\eta}_{0}^{b_{0}}\right](\dot{\gamma})\left(X_{b_{0}}^{0}\right)^{h^{1}} .
\end{aligned}
$$

Since $\left[\mathcal{D}, \mathcal{E}^{j}\right] \subsetneq\left[\mathcal{D}^{j+1}, \mathcal{D}^{j+1}\right] \subsetneq \mathcal{D}^{j+2}$, we have $\left[\widetilde{X}_{b_{0}}^{0}, \widetilde{X}_{a_{j}}^{j}\right] \in \Gamma\left(\mathcal{D}^{j+2}\right)$, and so $\left[\left(\widetilde{X}_{b_{0}}^{0}\right)^{h^{1}},\left(\widetilde{X}_{a_{j}}^{j}\right)^{h^{j+1}}\right] \in \Gamma\left(\mathcal{V} \oplus \mathcal{H}^{j+2}\right)$. Thus

$$
\begin{aligned}
£_{\Xi}\left(X_{a_{j}}^{j}\right)^{h^{j+1}}=\dot{\gamma}^{b_{0}} & \left(\mathscr{V}\left(\left[\left(\widetilde{X}_{b_{0}}^{0}\right)^{h^{1}},\left(\widetilde{X}_{a_{j}}^{j}\right)^{h^{j+1}}\right]\right)\right. \\
& +\left(\mathscr{P}_{j+1}-\mathscr{V}\right)\left(\left[\left(\widetilde{X}_{b_{0}}^{0}\right)^{h^{1}},\left(\widetilde{X}_{a_{j}}^{j}\right)^{h^{j+1}}\right]\right) \\
& \left.+\mathscr{Q}_{j+1}\left(\left[\left(\widetilde{X}_{b_{0}}^{0}\right)^{h^{1}},\left(\widetilde{X}_{a_{j}}^{j}\right)^{h^{j+1}}\right]\right)\right)(\dot{\gamma}) \\
& -\left(\widetilde{X}_{a_{j}}^{j}\right)^{h^{j+1}}\left[\bar{\eta}_{0}^{b_{0}}\right](\dot{\gamma})\left(X_{b_{0}}^{0}\right)^{h^{1}} .
\end{aligned}
$$

Consider the last term. Let $Z \in \Gamma(\mathcal{D})$ be an extension of $\dot{\gamma}$ to an open neighbourhood of $\gamma$; then

$$
\begin{aligned}
\left(\widetilde{X}_{a_{j}}^{j}\right)^{h^{j+1}}\left[\bar{\eta}_{0}^{b_{0}}\right](\dot{\gamma}) X_{b_{0}}^{0}= & \left\langle\nabla_{\widetilde{X}_{a_{j}}^{j}}^{j+1} \eta_{0}^{b_{0}}, Z\right\rangle(\gamma) X_{b_{0}}^{0} \\
= & \widetilde{X}_{a_{j}}^{j}\left[\left\langle\eta_{0}^{b_{0}}, Z\right\rangle\right](\gamma) X_{b_{0}}^{0}-\left\langle\eta_{0}^{b_{0}}, \nabla_{\widetilde{X}_{a_{j}}^{j}}^{j+1} Z\right\rangle(\gamma) X_{b_{0}}^{0} \\
= & \widetilde{X}_{a_{j}}^{j}\left[z^{b_{0}}\right](\gamma) X_{b_{0}}^{0}-\left\langle\eta_{0}^{b_{0}}, \widetilde{X}_{a_{j}}^{j}\left[z^{c_{0}}\right](\gamma) \widetilde{X}_{c_{0}}^{0}\right\rangle(\gamma) X_{b_{0}}^{0} \\
& \quad-\left\langle\eta_{0}^{b_{0}}, z^{c_{0}} \nabla_{\widetilde{X}_{a_{j}}^{j}+1}^{\left.j+\widetilde{X}_{c_{0}}^{0}\right\rangle(\gamma) X_{b_{0}}^{0}}\right. \\
= & -\left\langle\eta_{0}^{b_{0}}, z^{c_{0}} \nabla_{\widetilde{X}_{a_{j}}^{j}}^{j+1} \widetilde{X}_{c_{0}}^{0}\right\rangle(\gamma) X_{b_{0}}^{0} \\
= & -\dot{\gamma}^{c_{0}} \nabla_{\widetilde{X}_{a_{j}}^{j}+1}^{j+1} \widetilde{X}_{c_{0}}^{0}(\gamma) .
\end{aligned}
$$

Hence

$$
\begin{aligned}
£_{\Xi}\left(X_{a_{j}}^{j}\right)^{h^{j+1}}=\dot{\gamma}^{b_{0}} & \left(\mathscr{V}\left(\left[\left(\widetilde{X}_{b_{0}}^{0}\right)^{h^{1}},\left(\widetilde{X}_{a_{j}}^{j}\right)^{h^{j+1}}\right]\right)\right. \\
& +\left(\mathscr{P}_{j+1}-\mathscr{V}\right)\left(\left[\left(\widetilde{X}_{b_{0}}^{0}\right)^{h^{1}},\left(\widetilde{X}_{a_{j}}^{j}\right)^{h^{j+1}}\right]\right) \\
& \left.+\mathscr{Q}_{j+1}\left(\left[\left(\widetilde{X}_{b_{0}}^{0}\right)^{h^{1}},\left(\widetilde{X}_{a_{j}}^{j}\right)^{h^{j+1}}\right]\right)\right)(\dot{\gamma}) \\
& +h^{1}\left(\dot{\gamma}, \dot{\gamma}^{b_{0}} \nabla_{\widetilde{X}_{a_{j}}^{j}}^{j+1} \widetilde{X}_{b_{0}}^{0}(\gamma)\right) .
\end{aligned}
$$

Suppose that $j<N-1$; then

$$
\begin{aligned}
£_{\Xi}\left(X_{a_{j}}^{j}\right)^{h^{j+1}}= & \dot{\gamma}^{b_{0}}\left(\mathscr{V}\left(\left[\left(\widetilde{X}_{b_{0}}^{0}\right)^{h^{1}},\left(\widetilde{X}_{a_{j}}^{j}\right)^{h^{j+1}}\right]\right)+\mathscr{P}_{j+1}\left(\left[\widetilde{X}_{b_{0}}^{0}, \widetilde{X}_{a_{j}}^{j}\right]\right)^{h^{j+1}}\right. \\
& \left.+\mathscr{Q}_{j+1}\left(\left[\widetilde{X}_{b_{0}}^{0}, \widetilde{X}_{a_{j}}^{j}\right]\right)^{h^{j+2}}\right)(\dot{\gamma})+h^{1}\left(\dot{\gamma}, \dot{\gamma}^{b_{0}} \nabla_{\widetilde{X}_{a_{j}}^{j+1}}^{j+1} \widetilde{X}_{b_{0}}^{0}(\gamma)\right) \\
= & \dot{\gamma}^{b_{0}} \mathscr{V}\left(\left[\left(\widetilde{X}_{b_{0}}^{0}\right)^{h^{1}},\left(\widetilde{X}_{a_{j}}^{j}\right)^{h^{j+1}}\right]\right)(\dot{\gamma})+h^{1}\left(\dot{\gamma}, \dot{\gamma}^{b_{0}} \nabla_{\widetilde{X}_{a_{j}}^{j}}^{j+1} \widetilde{X}_{b_{0}}^{0}(\gamma)\right) \\
& +h^{j+1}\left(\dot{\gamma}, \dot{\gamma}^{b_{0}} \mathscr{P}_{j+1}\left(\left[\widetilde{X}_{b_{0}}^{0}, \widetilde{X}_{a_{j}}^{j}\right]\right)(\gamma)\right) \\
& +h^{j+2}\left(\dot{\gamma}, \dot{\gamma}^{b_{0}} \mathscr{Q}_{j+1}\left(\left[\widetilde{X}_{b_{0}}^{0}, \widetilde{X}_{a_{j}}^{j}\right]\right)(\gamma)\right) .
\end{aligned}
$$


The $h^{j+2}$-term is

$$
\begin{aligned}
h^{j+2}\left(\dot{\gamma}, \dot{\gamma}^{b_{0}} \mathscr{Q}_{j+1}\left(\left[\widetilde{X}_{b_{0}}^{0}, \widetilde{X}_{a_{j}}^{j}\right]\right)(\gamma)\right) & =h^{j+2}\left(\dot{\gamma}, \dot{\gamma}^{b_{0}} £_{X_{b_{0}}^{0}}^{\mathscr{Q}_{j+1}} X_{a_{j}}^{j}\right) \\
& =h^{j+2}\left(\dot{\gamma}, £_{\dot{\gamma}}^{\mathscr{Q}_{j+1}} X_{a_{j}}^{j}\right) .
\end{aligned}
$$

Consider the $h^{j+1}$-term; we have

$$
\begin{aligned}
& \dot{\gamma}^{b_{0}} \mathscr{P}_{j+1}\left(\left[\widetilde{X}_{b_{0}}^{0}, \widetilde{X}_{a_{j}}^{j}\right]\right)(\gamma)=\dot{\gamma}^{b_{0}}\left(\mathscr{P} \oplus \mathscr{Q}_{1} \oplus \cdots \oplus \mathscr{Q}_{j}\right)\left(\left[\widetilde{X}_{b_{0}}^{0}, \widetilde{X}_{a_{j}}^{j}\right]\right)(\gamma) \\
& =\dot{\gamma}^{b_{0}} \llbracket \widetilde{X}_{b_{0}}^{0}, \tilde{X}_{a_{j}}^{j} \rrbracket(\gamma)+\sum_{k=1}^{j} \dot{\gamma}^{b_{0}} \mathscr{Q}_{k}\left(\left[\widetilde{X}_{b_{0}}^{0}, \tilde{X}_{a_{j}}^{j}\right]\right)(\gamma) \\
& =\dot{\gamma}^{b_{0}}\left(K^{j}\left(\Theta_{j}\left(\widetilde{X}_{a_{j}}^{j}\right)\right) \widetilde{X}_{b_{0}}^{0}(\gamma)-\nabla_{\widetilde{X}_{a_{j}}^{j}}^{j+1} \widetilde{X}_{b_{0}}^{0}(\gamma)\right) \\
& +\sum_{k=1}^{j} £_{\dot{\gamma}}^{\mathscr{Q}^{k}} X_{a_{j}}^{j} \\
& =K^{j}\left(\Theta_{j}\left(X_{a_{j}}^{j}\right)\right) \dot{\gamma}-\dot{\gamma}^{b_{0}} \nabla_{\widetilde{X}_{a_{j}}^{j}}^{j+1} \widetilde{X}_{b_{0}}^{0}(\gamma)+\sum_{k=1}^{j} £_{\dot{\gamma}}^{\mathscr{Q}_{k}} X_{a_{j}}^{j} .
\end{aligned}
$$

By definition, $X_{a_{j}}^{j}$ is $£^{\mathscr{Q}_{j}}$-parallel along $\gamma$, i.e., $£_{\dot{\gamma}}^{\mathscr{Q}_{j}} X_{a_{j}}^{j}=0$. Accordingly, the $h^{j+1}$-term in $£_{\Xi}\left(X_{a_{j}}^{j}\right)^{h^{j+1}}$ is given by

$$
\begin{cases}h^{1}\left(\dot{\gamma}, K^{j}\left(\Theta_{j}\left(X_{a_{j}}^{j}\right)\right) \dot{\gamma}\right) & \text { if } j=1 \\ h^{1}\left(\dot{\gamma}, K^{j}\left(\Theta_{j}\left(X_{a_{j}}^{j}\right)\right) \dot{\gamma}\right)+\sum_{k=1}^{j-1} h^{k+1}\left(\dot{\gamma}, £_{\dot{\gamma}}^{\mathscr{Q}_{k}} X_{a_{j}}^{j}\right) & \text { if } j>1 .\end{cases}
$$

For the vertical term in $\Xi_{\Xi}\left(X_{a_{j}}^{j}\right)^{h^{j+1}}$, we have

$$
\begin{aligned}
& \dot{\gamma}^{b_{0}} \mathscr{V}\left(\left[\left(\widetilde{X}_{b_{0}}^{0}\right)^{h^{1}},\left(\widetilde{X}_{a_{j}}^{j}\right)^{h^{j+1}}\right]\right)(\dot{\gamma})=-\dot{\gamma}^{b_{0}} v\left(\dot{\gamma}, K^{j+1}\left(\widetilde{X}_{b_{0}}^{0} \wedge \widetilde{X}_{a_{j}}^{j}\right) \dot{\gamma}\right) \\
& =v\left(\dot{\gamma}, K^{j+1}\left(X_{a_{j}}^{j} \wedge \dot{\gamma}\right) \dot{\gamma}\right) \text {. }
\end{aligned}
$$

Hence, for $j=1$, we get

$$
£_{\Xi}\left(X_{a_{1}}^{1}\right)^{h^{2}}=v\left(\dot{\gamma}, K^{2}\left(X_{a_{1}}^{1} \wedge \dot{\gamma}\right) \dot{\gamma}\right)+h^{1}\left(\dot{\gamma}, K^{1}\left(\Theta_{1}\left(X_{a_{1}}^{1}\right)\right) \dot{\gamma}\right)+h^{3}\left(\dot{\gamma}, £_{\dot{\gamma}}^{\mathscr{Q}_{2}} X_{a_{1}}^{1}\right) .
$$

Likewise, for $j>1$, we get

$$
\begin{aligned}
£_{\Xi}\left(X_{a_{j}}^{j}\right)^{h^{j+1}}=v & \left(\dot{\gamma}, K^{j+1}\left(X_{a_{j}}^{j} \wedge \dot{\gamma}\right) \dot{\gamma}\right)+h^{1}\left(\dot{\gamma}, K^{j}\left(\Theta_{j}\left(X_{a_{j}}^{j}\right)\right) \dot{\gamma}\right) \\
& +\sum_{k=1}^{j-1} h^{k+1}\left(\dot{\gamma}, £_{\dot{\gamma}}^{\mathscr{Q}_{k}} X_{a_{j}}^{j}\right)+h^{j+2}\left(\dot{\gamma}, £_{\dot{\gamma}}^{\mathscr{Q}_{j+1}} X_{a_{j}}^{j}\right) .
\end{aligned}
$$

This yields the first and second parts of (4.9), respectively. Suppose now that $j=N-1$; a similar argument to that above then yields

$$
\begin{gathered}
£_{\Xi}\left(X_{a_{N-1}}^{N-1}\right)^{h^{N}}=v\left(\dot{\gamma}, K^{N}\left(X_{a_{N-1}}^{N-1} \wedge \dot{\gamma}\right) \dot{\gamma}\right)+h^{1}\left(\dot{\gamma}, K^{N-1}\left(\Theta_{N-1}\left(X_{a_{N-1}}^{N-1}\right)\right) \dot{\gamma}\right) \\
+\sum_{k=1}^{N-2} h^{k+1}\left(\dot{\gamma}, £_{\dot{\gamma}}^{\mathscr{Q}_{k}} X_{a_{N-1}^{N-1}}^{N-1} .\right.
\end{gathered}
$$

Hence we obtain the last part of (4.9).

In order to state the equation for restricted Jacobi fields in a form comparable to that of Theorem 3.1, we shall introduce certain restricted connections, as well as tensors that will play the rôle of the Jacobi operators. Let $D^{1}=\nabla$ and let $D^{i+1}: \Gamma(\mathcal{D}) \times \Gamma\left(\mathcal{D}^{i+1}\right) \rightarrow \Gamma\left(\mathcal{D}^{i+1}\right)$ be the $\mathcal{D}$-connection on $\mathcal{D}^{i+1}$ given by

$$
D_{U}^{i+1} X=\nabla_{X}^{i+1} U-\mathscr{P}_{i+1}([X, U]), \quad U \in \Gamma(\mathcal{D}), X \in \Gamma\left(\mathcal{D}^{i+1}\right) .
$$


Similarly, if $U \in \Gamma(\mathcal{D})$, then let $\Psi_{U}^{1}=\Psi_{U}$, and let $\Psi_{U}^{i+1}: \mathcal{D}^{i+1} \rightarrow \mathcal{D}$ be the tensor given by

$$
\Psi_{U}^{i+1}(X)=\sum_{j=0}^{i-1} K^{j+1}\left(X_{j} \wedge U\right) U+R^{i+1}\left(X_{i} \wedge U\right) U
$$

for $X \in \Gamma\left(\mathcal{D}^{i+1}\right)$, where $X_{0}=\mathscr{P}(X)$ and $X_{k}(X)=\mathscr{Q}_{k}(X), k=1, \ldots, i$.

Theorem 4.1. If $V$ is an $\mathcal{H}^{i+1}$-Jacobi field along $\dot{\gamma}$, then the $\mathcal{D}^{i+1}$-variation vector field (along $\gamma$ ) $J=T \pi \cdot V \in \Gamma\left(\gamma^{*} \mathcal{D}^{i+1}\right.$ ) satisfies

$$
\nabla_{\dot{\gamma}} D_{\dot{\gamma}}^{i+1} J+\Psi_{\dot{\gamma}}^{i+1}(J)=0 \text { and } £_{\dot{\gamma}}^{\mathscr{Q}} J=0 .
$$

(As $£_{\dot{\gamma}}^{\mathcal{Q}} J=0$, it follows that $D_{\dot{\gamma}}^{i+1} J \in \Gamma\left(\gamma^{*} \mathcal{D}\right)$.) Conversely, if $J \in \Gamma\left(\gamma^{*} \mathcal{D}^{i+1}\right)$ satisfies (4.13), then there exists a unique $\mathcal{H}^{i+1}$-Jacobi field $V$ along $\dot{\gamma}$ such that $J=T \pi \cdot V$.

Proof. Let $V=x^{a_{0}}\left(X_{a_{0}}^{0}\right)^{v}+y_{0}^{a_{0}}\left(X_{a_{0}}^{0}\right)^{h}+\sum_{j=1}^{i} y_{j}^{a_{j}}\left(X_{a_{j}}^{j}\right)^{h^{j+1}}$ for $x^{a_{0}}, y_{0}^{a_{0}}, y_{j}^{a_{j}} \in \mathcal{C}^{\infty}([0, T])$, and let $J=T \pi \cdot V=$ $y_{0}^{a_{0}} X_{a_{0}}^{0}+\sum_{j=1}^{i} y_{j}^{a_{j}} X_{a_{j}}^{j}$; then $\mathscr{P}(J)=y_{0}^{a_{0}} X_{a_{0}}^{0}$ and $\mathscr{Q}_{j}(J)=y_{j}^{a_{j}} X_{a_{j}}^{j}$ for $j=1, \ldots, i$. Let $\widetilde{J} \in \Gamma\left(\mathcal{D}^{i+1}\right)$ and $Z \in \Gamma(\mathcal{D})$ be extensions of $J$ and $\dot{\gamma}$, respectively, to an open neighbourhood of $\gamma$. We have

$$
\begin{aligned}
& £_{\Xi} V=\dot{x}^{a_{0}}\left(X_{a_{0}}^{0}\right)^{v}+x^{a_{0}} £_{\Xi}\left(X_{a_{0}}^{0}\right)^{v}+\dot{y}_{0}^{a_{0}}\left(X_{a_{0}}^{0}\right)^{h^{1}}+y_{0}^{a_{0}} £_{\Xi}\left(X_{a_{0}}^{0}\right)^{h^{1}} \\
& +\sum_{j=1}^{i}\left(\dot{y}_{j}^{a_{j}}\left(X_{a_{j}}^{j}\right)^{h^{j+1}}+y_{j}^{a_{j}} £_{\Xi}\left(X_{a_{j}}^{j}\right)^{h^{j+1}}\right) .
\end{aligned}
$$

We shall prove only the case when $N>2$ and $i=N-1$; the remaining cases (one needs to consider $N=2 ; N>2$, $i=1$; and $N>2,1<i<N-1$ ) are proved similarly. Hence, taking $N>2$ and $i=N-1$, we may substitute for $£_{\Xi}\left(X_{a_{0}}^{0}\right)^{v}, £_{\Xi}\left(X_{a_{0}}^{0}\right)^{h^{1}}$, and $£_{\Xi}\left(X_{a_{j}}^{j}\right)^{h^{j+1}}$ using the equations (4.5) and (4.9) in Proposition 4.3. After a lengthy (and somewhat tedious) calculation, we obtain

$$
\begin{aligned}
£_{\Xi} V=v & \left(\dot{\gamma}, \dot{x}^{a_{0}} X_{a_{0}}^{0}+K^{1}(\mathscr{P}(J) \wedge \dot{\gamma}) \dot{\gamma}+\sum_{j=1}^{N-1} K^{j+1}\left(\mathscr{Q}_{j}(J) \wedge \dot{\gamma}\right) \dot{\gamma}\right) \\
& \left.+h\left(\dot{\gamma}, \dot{y}_{0}^{a_{0}}-x^{a_{0}}\right) X_{a_{0}}^{0}+\sum_{j=1}^{N-1} K^{j}\left(\Theta_{j}\left(\mathscr{Q}_{j}(J)\right)\right) \dot{\gamma}\right) \\
& +h^{2}\left(\dot{\gamma}, \dot{y}_{1}^{a_{1}} X_{a_{1}}^{1}+£_{\dot{\gamma}}^{\mathcal{Q}_{1}} \mathscr{P}(J)+\sum_{k=2}^{N-1} £_{\dot{\gamma}}^{\mathcal{Q}_{1}} \mathscr{Q}_{k}(J)\right) \\
& +\sum_{j=2}^{N-2} h^{j+1}\left(\dot{\gamma}, \dot{y}_{j}^{a_{j}} X_{a_{j}}^{j}+£_{\dot{\gamma}}^{\mathscr{Q}_{j}} \mathscr{Q}_{j-1}(J)+\sum_{k=j+1}^{N-1} £_{\dot{\gamma}}^{\mathscr{Q}_{j}} \mathscr{Q}_{k}(J)\right) \\
& +h^{N}\left(\dot{\gamma}_{,} \dot{y}_{N-1}^{a_{N-1}} X_{a_{N-1}}^{N-1}+£_{\dot{\gamma}}^{\mathscr{Q}_{N-1}} \mathscr{Q}_{N-2}(J)\right) .
\end{aligned}
$$

(If $N=3$, then the $h^{j+1}$ terms fall away.) Suppose that $V$ is an $\mathcal{H}^{N}$-Jacobi field along $\dot{\gamma}$, i.e., $£_{\Xi} V=0$; then the components of $J$ satisfy

$$
\begin{aligned}
& \dot{x}^{a_{0}} X_{a_{0}}^{0}=-K^{1}(\mathscr{P}(J) \wedge \dot{\gamma}) \dot{\gamma}-\sum_{j=1}^{N-1} K^{j+1}\left(\mathscr{Q}_{j}(J) \wedge \dot{\gamma}\right) \dot{\gamma} \\
&\left(\dot{y}_{0}^{a_{0}}-x^{a_{0}}\right) X_{a_{0}}^{0}=-\sum_{j=1}^{N-1} K^{j}\left(\Theta_{j}\left(\mathscr{Q}_{j}(J)\right) \dot{\gamma}\right. \\
& \dot{y}_{1}^{a_{1}} X_{a_{1}}^{1}=-£_{\dot{\gamma}}^{\mathscr{Q}_{1}}\left(\mathscr{P} \oplus \mathscr{Q}_{2} \oplus \cdots \oplus \mathscr{Q}_{N-1}\right)(J) \\
& \dot{y}_{j}^{a_{j}} X_{a_{j}}^{j}=-£_{\dot{\gamma}}^{\mathscr{Q}_{j}}\left(\mathscr{Q}_{j-1} \oplus \mathscr{Q}_{j+1} \oplus \cdots \oplus \mathscr{Q}_{N-1}\right)(J) \\
& \quad \quad \text { for } j=2, \ldots, N-2 \\
& \dot{y}_{N-1}^{a_{N-1}} X_{a_{N-1}}^{N-1}=-£_{\dot{\gamma}}^{\mathscr{Q}_{N-1}} \mathscr{Q}_{N-2}(J) .
\end{aligned}
$$

(Equation (4.17) falls away when $N=3$.) We have $£_{\dot{\gamma}}^{\mathscr{Q}_{j}} \mathscr{Q}_{j}(J)=\dot{y}_{j}^{a_{j}} X_{a_{j}}^{j}$ for $j=1, \ldots, N-1$; furthermore, $\mathscr{P}_{N}(J)=J$. Consequently, equations (4.16), (4.17), and (4.18) yield

$$
£_{\dot{\gamma}}^{\mathscr{Q}_{1}} J=0 \quad \text { and } \quad £_{\dot{\gamma}}^{\mathscr{Q}_{j}}\left(\mathscr{Q}_{j-1} \oplus \cdots \oplus \mathscr{Q}_{N-1}\right)(J)=0,
$$


for $j=2, \ldots, N-1$. Since $[\mathcal{D}, \mathcal{D}] \subsetneq \mathcal{D}^{2}=\mathcal{D} \oplus \mathcal{E}^{1}$ and $\left[\mathcal{D}, \mathcal{E}^{j}\right] \subsetneq\left[\mathcal{D}^{j+1}, \mathcal{D}^{j+1}\right] \subsetneq \mathcal{D}^{j+2}=\mathcal{D}^{j+1} \oplus \mathcal{E}^{j+1}$, we get

$$
\begin{aligned}
£_{\dot{\gamma}}^{\mathscr{Q}_{j}} J & =\mathscr{Q}_{j}(\underbrace{[Z, \mathscr{P}(\widetilde{J})]}_{\in \mathcal{D}^{2}})(\gamma)+\mathscr{Q}_{j}(\underbrace{\left[Z, \mathscr{Q}_{1}(\widetilde{J})\right.}_{\in \mathcal{D}^{3}})(\gamma)+\cdots+\mathscr{Q}_{j}(\underbrace{\left[Z, \mathscr{Q}_{N-1}(\widetilde{J})\right]}_{\in \mathcal{D}^{N+1}=\mathcal{D}^{N}})(\gamma) \\
& =\mathscr{Q}_{j}\left(\left[Z, \mathscr{Q}_{j-1}(\widetilde{J})\right]\right)(\gamma)+\cdots+\mathscr{Q}_{j}\left(\left[Z, \mathscr{Q}_{N-1}(\widetilde{J})\right]\right)(\gamma) \\
& =£_{\dot{\gamma}}^{\mathscr{Q}_{j}}\left(\mathscr{Q}_{j-1} \oplus \cdots \oplus \mathscr{Q}_{N-1}\right)(J) \\
& =0,
\end{aligned}
$$

for $j=2, \ldots, N-1$. Since we already have $£_{\dot{\gamma}}^{\mathscr{Q}_{1}} J=0$, it follows that $£_{\dot{\gamma}}^{\mathscr{Q}} J=0$. Consider now equation (4.15). By Lemma 2.1, we have

$$
\begin{aligned}
\sum_{j=1}^{N-1} K^{j}\left(\Theta_{j}\left(\mathscr{Q}_{j}(J)\right)\right) \dot{\gamma} & =\nabla_{J}^{N} \dot{\gamma}-\nabla_{\mathscr{P}(J)} \dot{\gamma}-\llbracket \mathscr{Q}(\widetilde{J}), Z \rrbracket(\gamma) \\
& =\nabla_{J}^{N} \dot{\gamma}-\nabla_{\dot{\gamma}} \mathscr{P}(J)+\llbracket Z, \widetilde{J} \rrbracket(\gamma) .
\end{aligned}
$$

Since $\nabla_{\dot{\gamma}} \mathscr{P}(J)=\dot{y}_{0}^{a_{0}} X_{a_{0}}^{0}$, we get

$$
\begin{aligned}
x^{a_{0}} X_{a_{0}}^{0} & =\dot{y}_{0}^{a_{0}} X_{a_{0}}^{0}+\sum_{j=1}^{N-1} K^{j}\left(\Theta_{j}\left(\mathscr{Q}_{j}(J)\right)\right) \dot{\gamma} \\
& =\nabla_{\dot{\gamma}} \mathscr{P}(J)+\nabla_{J}^{N} \dot{\gamma}-\nabla_{\dot{\gamma}} \mathscr{P}(J)+\llbracket Z, \widetilde{J} \rrbracket(\gamma)=\nabla_{J}^{N} \dot{\gamma}-\llbracket \widetilde{J}, Z \rrbracket(\gamma)=D_{\dot{\gamma}}^{N} J .
\end{aligned}
$$

(In the last step we use the fact that $£_{\dot{\gamma}}^{\mathscr{Q}} J=0$.) Hence, from (4.14), we get

$$
\nabla_{\dot{\gamma}} D_{\dot{\gamma}}^{N} J=\dot{x}^{a_{0}} X_{a_{0}}^{0}=-K^{1}(\mathscr{P}(J) \wedge \dot{\gamma}) \dot{\gamma}-\sum_{j=1}^{N-1} K^{j+1}\left(\mathscr{Q}_{j}(J) \wedge \dot{\gamma}\right) \dot{\gamma} .
$$

Since $C^{N}$ vanishes identically, it is trivially true that $C^{N}(\mathscr{Q}(J) \wedge \dot{\gamma}) \dot{\gamma}=0$. (For the other cases, one must use $£_{\dot{\gamma}}^{\mathscr{Q}} J=0$, together with that fact that $\left.C^{i+1}\right|_{\mathcal{D}^{i} \wedge \mathcal{D}^{i} \otimes \mathcal{D}}=0$, to show that $C^{i+1}\left(\mathscr{Q}_{i}(J) \wedge \dot{\gamma}\right) \dot{\gamma}=0$.) Hence

$$
\nabla_{\dot{\gamma}} D_{\dot{\gamma}}^{N} J=-\Psi_{\dot{\gamma}}^{i+1}(J) \text {. }
$$

Therefore, if $V$ is an $\mathcal{H}^{N}$-Jacobi field along $\dot{\gamma}$, then $J=T \pi \cdot V$ satisfies (4.13).

Conversely, suppose that $J=y_{0}^{a_{0}} X_{a_{0}}^{0}+\sum_{j=1}^{i} y_{j}^{a_{j}} X_{a_{j}}^{j} \in \Gamma\left(\gamma^{*} \mathcal{D}^{i+1}\right)$ satisfies (4.13). Let $V=x^{a_{0}}\left(X_{a_{0}}^{0}\right)^{v}+$ $y_{0}^{a_{0}}\left(X_{a_{0}}^{0}\right)^{h}+\sum_{j=1}^{i} y_{j}^{a_{j}}\left(X_{a_{j}}^{j}\right)^{h^{j+1}} \in \Gamma\left(\dot{\gamma}^{*}\left(\mathcal{V} \oplus \mathcal{H}^{i+1}\right)\right)$, where

$$
x^{a_{0}}=\dot{y}^{a_{0}}-\sum_{j=1}^{i}\left\langle\nu_{0}^{a_{0}}, K^{j}\left(\Theta_{j}\left(\mathscr{Q}_{j}(J)\right)\right) \dot{\gamma}\right\rangle,
$$

where $\left(\nu_{0}^{a_{0}}\right)$ is dual to $\left(X_{a_{0}}^{0}\right)$. It should be clear that $V$ satisfies $£_{\Xi} V=0$, i.e., $V$ is an $\mathcal{H}^{i+1}$-Jacobi field along $\dot{\gamma}$. It remains to prove uniqueness. Let $\bar{V}=\bar{x}^{a_{0}}\left(X_{a_{0}}^{0}\right)^{v}+\bar{y}_{0}^{a_{0}}\left(X_{a_{0}}^{0}\right)^{h}+\sum_{j=1}^{i} \bar{y}_{j}^{a_{j}}\left(X_{a_{j}}^{j}\right)^{h^{j+1}}$ be another $\mathcal{H}^{i+1}$-Jacobi field along $\dot{\gamma}$ such that $T \pi \cdot \bar{V}=J$; then

$$
\bar{y}_{0}^{a_{0}} X_{a_{0}}^{0}+\sum_{j=1}^{i} \bar{y}_{j}^{a_{j}} X_{a_{j}}^{j}=T \pi \cdot \bar{V}=J=y_{0}^{a_{0}} X_{a_{0}}^{0}+\sum_{j=1}^{i} y_{j}^{a_{j}} X_{a_{j}}^{j} .
$$

That is, $\bar{y}_{0}^{a_{0}}=y_{0}^{a_{0}}$ and $\bar{y}_{j}^{a_{j}}=y_{j}^{a_{j}}$ for each $j=1, \ldots, i$. Furthermore, since $£_{\Xi} \bar{V}=0$, we have $\left(\dot{y}^{a_{0}}-\bar{x}^{a_{0}}\right) X_{a_{0}}^{0}=$ $\sum_{j=1}^{i} K^{j}\left(\Theta_{j}\left(\mathscr{Q}_{j}(J)\right)\right) \dot{\gamma}$; it follows that $\bar{x}^{a_{0}}=x^{a_{0}}$. Thus $\bar{V}=V$, and so $V$ is unique.

A vector field $J \in \Gamma\left(\gamma^{*} \mathcal{D}^{i+1}\right)$ is called a $\mathcal{D}^{i+1}$-restricted Jacobi field along $\gamma\left(\right.$ or simply a $\mathcal{D}^{i+1}$-Jacobi field along $\gamma$ ) if

$$
\nabla_{\dot{\gamma}} D_{\dot{\gamma}}^{i+1} J+\Psi_{\dot{\gamma}}^{i+1}(J)=0 \text { and } £_{\dot{\gamma}}^{\mathcal{Q}} J=0 .
$$

For completeness, we also define a $\mathcal{D}^{1}$-Jacobi field along $\gamma$ to be a nonholonomic Jacobi field along $\gamma$. It should be clear from Theorem 4.1 that there exists a one-to-one correspondence between $\mathcal{D}^{i+1}$-Jacobi fields along $\gamma$ and $\mathcal{H}^{i+1}$-Jacobi fields along $\dot{\gamma}$.

Remark 4.1. So far as the authors of this paper are aware, the significance of the connections $D^{2}, \ldots, D^{N}$ and the operators $\Psi_{U}^{2}, \ldots, \Psi_{U}^{N}$ is not known. In this vein, it would be of interest to learn if they appear in other contexts. 


\section{Some remarks on existence}

We conclude the paper by discussing the existence of restricted Jacobi fields. It is well known that, in the Riemannian case, there exists a unique Jacobi field $J$ along a geodesic $\gamma$ for initial conditions $J(0)$ and $\nabla_{\dot{\gamma}} J(0)$. It should be clear that, if a restricted Jacobi field does exist, then it is uniquely determined by its initial conditions; however, it turns out that there does not generally exist a restricted Jacobi field for all possible initial conditions (see Corollary 5.1 below, and the subsequent comments).

Let $\left(\mathrm{M}, \mathcal{D}, \mathcal{D}^{\perp}, \mathrm{g}\right.$ ) be a Wagner structure (with degree of nonholonomy $\left.N\right)$, and let $\gamma:[0, T] \rightarrow \mathrm{M}$ be a nonholonomic geodesic. Denote by $\mathcal{J}_{\gamma}^{k}$ the space of all $\mathcal{D}^{k}$-Jacobi fields along $\gamma($ for $k=1, \ldots, N)$; by linearity of the conditions (3.12) and (4.24) in $J$, it follows that each $\mathcal{J}_{\gamma}^{k}$ is a vector space. We have that $\dot{\gamma}$ is itself a $\mathcal{D}^{1}$-Jacobi field along $\gamma$; let $\mathcal{T}_{\gamma}$ denote the subspace of tangential Jacobi fields (i.e., $\mathcal{D}^{1}$-Jacobi fields parallel to $\dot{\gamma}$ ):

$$
\mathcal{T}_{\gamma}=\left\{J \in \mathcal{J}_{\gamma}^{1}: J=f \dot{\gamma} \text { for some } f \in \mathcal{C}^{\infty}([0, T])\right\} .
$$

\section{Proposition 5.1.}

1. $J \in \mathcal{T}_{\gamma}$ if and only if $J(t)=\left(k_{0}+k_{1} t\right) \dot{\gamma}(t)$ for some $k_{0}, k_{1} \in \mathbb{R}$.

2. We have $\mathcal{T}_{\gamma} \subseteq \mathcal{J}_{\gamma}^{1} \subseteq \cdots \subseteq \mathcal{J}_{\gamma}^{N}$.

Proof. (1.) Let $J=f \dot{\gamma}$ be a $\mathcal{D}^{1}$-Jacobi field, for some $f \in \mathcal{C}^{\infty}([0, T])$; then

$$
\nabla_{\dot{\gamma}} \nabla_{\dot{\gamma}} J+\Psi_{\dot{\gamma}}(J)=0 \quad \Longleftrightarrow \nabla_{\dot{\gamma}} \nabla_{\dot{\gamma}} J=0 \quad \Longleftrightarrow \quad \ddot{f}=0 .
$$

Hence every tangential Jacobi field takes precisely the form $t \mapsto\left(k_{0}+k_{1} t\right) \dot{\gamma}(t)$ for some $k_{0}, k_{1} \in \mathbb{R}$.

(2.) We have, by definition, $\mathcal{T}_{\gamma} \subseteq \mathcal{J}_{\gamma}^{1}$. Let $1 \leq i \leq N-1$ and let $J \in \mathcal{J}_{\gamma}^{i}$; we claim that $J \in \mathcal{J}_{\gamma}^{i+1}$. Indeed, we have $D_{\dot{\gamma}}^{i+1} J=D_{\dot{\gamma}}^{i} J-£_{\dot{\gamma}}^{\mathcal{Q}_{i}} J=D_{\dot{\gamma}}^{i} J\left(\right.$ since $\left.£_{\dot{\gamma}}^{\mathscr{Q}} J=0\right)$ ). Hence

$$
\nabla_{\dot{\gamma}} D_{\dot{\gamma}}^{i+1} J+\Psi_{\dot{\gamma}}^{i+1}(J)=\nabla_{\dot{\gamma}} D_{\dot{\gamma}}^{i} J+\Psi_{\dot{\gamma}}^{i+1}(J) .
$$

Since $J \in \Gamma\left(\gamma^{*} \mathcal{D}^{i}\right)$, it follows that $\mathscr{Q}_{i}(J)=0$, and so $R^{i+1}\left(\mathscr{Q}_{i}(J) \wedge \dot{\gamma}\right) \dot{\gamma}=0$. Suppose that $i=1$; then $\mathscr{P}(J)=J$, $D_{\dot{\gamma}}^{1} J=\nabla_{\dot{\gamma}} J$, and the condition $£_{\dot{\gamma}}^{\mathscr{Q}} J=0$ implies that $C^{1}(J, \dot{\gamma}) \dot{\gamma}=0$. Hence $\Psi_{\dot{\gamma}}^{2}(J)=\Psi_{\dot{\gamma}}^{1}(J)$, and so $\nabla_{\dot{\gamma}} D_{\dot{\gamma}}^{2} J+$ $\Psi_{\dot{\gamma}}^{2}(J)=\nabla_{\dot{\gamma}} D_{\dot{\gamma}}^{1} J+\Psi_{\dot{\gamma}}^{1}(J)=0$. That is, $J \in \mathcal{J}_{\gamma}^{2}$, whence $\mathcal{J}_{\gamma}^{1} \subseteq \mathcal{J}_{\gamma}^{2}$. Suppose now that $i>1$; then

$$
\begin{aligned}
& 0=£_{\dot{\gamma}}^{\mathscr{Q}_{i}} J \\
& =\mathscr{Q}_{i}(\underbrace{[Z, \mathscr{P}(\widetilde{J})]}_{\in \mathcal{D}^{2}=\mathcal{D} \oplus \mathcal{E}^{1}})(\gamma)+\mathscr{Q}_{i}(\underbrace{\left[Z, \mathscr{Q}_{1}(\widetilde{J})\right]}_{\in \mathcal{D}^{3}=\mathcal{D}^{2} \oplus \mathcal{E}^{2}})(\gamma)+\cdots+\mathscr{Q}_{i}(\underbrace{\left[Z, \mathscr{Q}_{i-1}(\widetilde{J})\right]}_{\in \mathcal{D}^{i+1}=\mathcal{D}^{i} \oplus \mathcal{E}^{i}})(\gamma) \\
& =\mathscr{Q}_{i}\left(\left[Z, \mathscr{Q}_{i-1}(\widetilde{J})\right]\right)(\gamma)=£_{\dot{\gamma}}^{\mathscr{Q}_{i}} \mathscr{Q}_{i-1}(J) .
\end{aligned}
$$

It follows that $C^{i}\left(\mathscr{Q}_{i-1}(J), \dot{\gamma}\right) \dot{\gamma}=0$, and so

$$
\begin{aligned}
\Psi_{\dot{\gamma}}^{i+1}(J) & =K^{1}(\mathscr{P}(J), \dot{\gamma}) \dot{\gamma}+\sum_{j=1}^{i-1} K^{j+1}\left(\mathscr{Q}_{j}(J), \dot{\gamma}\right) \dot{\gamma}+R^{i+1}\left(\mathscr{Q}_{i}(J), \dot{\gamma}\right) \dot{\gamma} \\
& =K^{1}(\mathscr{P}(J), \dot{\gamma}) \dot{\gamma}+\sum_{j=1}^{i-2} K^{j+1}\left(\mathscr{Q}_{j}(J), \dot{\gamma}\right) \dot{\gamma}+R^{i}\left(\mathscr{Q}_{i-1}(J), \dot{\gamma}\right) \dot{\gamma}=\Psi_{\dot{\gamma}}^{i}(J)
\end{aligned}
$$

Hence $\nabla_{\dot{\gamma}} D_{\dot{\gamma}}^{i+1} J+\Psi_{\dot{\gamma}}^{i+1}(J)=\nabla_{\dot{\gamma}} D_{\dot{\gamma}}^{i} J+\Psi_{\dot{\gamma}}^{i}(J)=0$, i.e., $J \in \mathcal{J}_{\gamma}^{i+1}$. Therefore, by induction, $\mathcal{J}_{\gamma}^{1} \subseteq \cdots \subseteq \mathcal{J}_{\gamma}^{N}$.

Proposition 5.2. If $\operatorname{ker} \Lambda_{i}=\{0\}$ for some $1 \leq i \leq N-1$, then $\mathcal{T}_{\gamma}=\mathcal{J}_{\gamma}^{1}=\cdots=\mathcal{J}_{\gamma}^{i}$.

Proof. Suppose that $\operatorname{ker} \Lambda_{i}=\{0\}$ for some $1 \leq i \leq N-1$, and let $J \in \mathcal{J}_{\gamma}^{i}$; then $\Lambda_{i}(\dot{\gamma} \wedge J)=£_{\dot{\gamma}}^{\mathcal{Q}_{i}} J=0$. Since $\operatorname{ker} \Lambda_{i}$ has trivial kernel, it follows that $\dot{\gamma} \wedge J=0$, i.e., $J=f \dot{\gamma}$ for some $f \in \mathcal{C}^{\infty}([0, T])$, and so $J \in \mathcal{T}_{\gamma}$. Thus $\mathcal{J}_{\gamma}^{i} \subseteq \mathcal{T}_{\gamma}$, whence $\mathcal{T}_{\gamma}=\mathcal{J}_{\gamma}^{1}=\cdots=\mathcal{J}_{\gamma}^{i}$.

Corollary 5.1. Suppose $\operatorname{dim} \mathrm{M}=3$.

1. We have $\mathcal{T}_{\gamma}=\mathcal{J}_{\gamma}^{1}$. 
2. Let $X_{q}, Y_{q} \in \mathcal{D}_{q}$, where $q=\gamma(0)$. There exists a nonholonomic Jacobi field $J$ along $\gamma$ such that $J(0)=X_{q}$ and $\nabla_{\dot{\gamma}} J(0)=Y_{q}$ if and only if $X_{q}, Y_{q} \in \operatorname{span}\{\dot{\gamma}(0)\}$.

Proof. (1.) Since $\operatorname{dim} \mathrm{M}=3$, we have $\operatorname{rank} \mathcal{D}=2$. Let $\left(X_{0}, X_{1}, X_{2}\right)$ be a frame on $\mathrm{M}$, where $\left(X_{1}, X_{2}\right)$ is a frame for $\mathcal{D}$, and let $c_{i j}^{k} \in \mathcal{C}^{\infty}(\mathrm{M})$ be the structure constants given by $\left[X_{i}, X_{j}\right]=c_{i j}^{k} X_{k}$. We have $\Lambda_{1}\left(X_{1} \wedge X_{2}\right)=\mathscr{Q}\left(\left[X_{1}, X_{2}\right]\right)=$ $c_{12}^{0} X_{0} \neq 0$. (If $c_{12}^{0}=0$, then $\mathcal{D}$ would be holonomic.) Hence, as $\Lambda_{1}$ is completely specified by $\Lambda_{1}\left(X_{1} \wedge X_{2}\right)$, it follows that $\operatorname{ker} \Lambda_{1}=\{0\}$. The result now follows from Proposition 5.2.

(2.) Let $J \in \mathcal{J}_{\gamma}^{1}$; then $J(t)=\left(k_{0}+k_{1} t\right) \dot{\gamma}(t)$ for some $k_{0}, k_{1} \in \mathbb{R}$. Hence $J(0)=k_{0} \dot{\gamma}(0)$ and $\nabla_{\dot{\gamma}} J(0)=k_{1} \dot{\gamma}(0)$, i.e., we have $J(0)=X_{q}, \nabla_{\dot{\gamma}} J(0)=Y_{q}$ if and only if $X_{q}, Y_{q} \in \operatorname{span}\{\dot{\gamma}(0)\}$.

So, by Corollary 5.1, there does not generally exist a nonholonomic Jacobi field along $\gamma$ for any given initial conditions. In fact, one can find nonholonomic Riemannian structures for which there do not exist $\mathcal{D}^{k}$-Jacobi fields (for any $1<k \leq N$ ) with arbitrary initial conditions. The problem of existence arises from the requirement that $£_{\dot{\gamma}}^{\mathscr{Q}} J=0$. It may be of interest to consider vector fields along $\gamma$ that satisfy the differential equation for a restricted Jacobi field, but without the foregoing requirement. Hence we make the following definition.

Fix $1 \leq k \leq N$. We shall call a vector field $J \in \Gamma\left(\gamma^{*} \mathcal{D}^{k}\right)$ a pre- $\mathcal{D}^{k}$-Jacobi field along $\gamma$ if

$$
\nabla_{\dot{\gamma}}\left(\nabla_{J}^{k} \dot{\gamma}+£_{\dot{\gamma}}^{\mathscr{P}} J\right)+\Psi_{\dot{\gamma}}^{k}(J)=0
$$

(Without the condition $£_{\dot{\gamma}}^{\mathscr{Q}} J=0$, we cannot write the first term above as $\nabla_{\dot{\gamma}} D_{\dot{\gamma}}^{k} J$; instead, we write it in an equivalent form.) The following result is easy to prove.

Proposition 5.3. Let $X_{q} \in \mathcal{D}_{q}$ and $Y_{q} \in \mathcal{D}_{q}^{i+1}$, where $q=\gamma(0)$. There exists a unique pre- $\mathcal{D}^{k}$-Jacobi field $J$ such that $J(0)=Y_{q}$ and $\nabla_{\dot{\gamma}} \mathscr{P}(J)(0)=X_{q}$.

Let $\mathcal{P}_{\gamma}^{k}$ be the vector space of pre- $\mathcal{D}^{k}$-Jacobi fields along $\gamma$. Clearly, we have $\mathcal{J}_{\gamma}^{k} \subseteq \mathcal{P}_{\gamma}^{k}$, and $\mathcal{T}_{\gamma} \subseteq \mathcal{P}_{\gamma}^{1} \subseteq \cdots \subseteq \mathcal{P}_{\gamma}^{N}$. The space of pre- $\mathcal{D}^{1}$-Jacobi fields, in particular, has properties similar to the space of Jacobi fields in Riemannian geometry.

\section{Proposition 5.4 (cf. [18]).}

1. Let $t \in[0, T]$; the maps

$$
\begin{array}{rlrl}
\mathcal{P}_{\gamma}^{1} & \rightarrow \mathcal{D}_{\gamma(t)} \times \mathcal{D}_{\gamma(t)}, & J \mapsto\left(J(t), \nabla_{\dot{\gamma}} J(t)\right) \\
\mathcal{P}_{\gamma}^{i+1} \rightarrow \mathcal{D}_{\gamma(t)}^{i+1} \times \mathcal{D}_{\gamma(t)}, & J \mapsto\left(J(t), \nabla_{\dot{\gamma}} \mathscr{P}(J)(t)\right)
\end{array}
$$

are isomorphisms. In particular, we have $\operatorname{dim} \mathcal{P}_{\gamma}^{1}=2 r_{0}$ and $\operatorname{dim} \mathcal{P}_{\gamma}^{i+1}=2 r_{0}+r_{1}+\cdots+r_{i}$.

2. $\mathcal{P}_{\gamma}^{1}$ admits a natural symplectic structure $\omega_{\gamma}$, given by

$$
\omega_{\gamma}(J, L)=\mathbf{g}\left(J, \nabla_{\dot{\gamma}} L\right)-\mathbf{g}\left(\nabla_{\dot{\gamma}} J, L\right), \quad J, L \in \mathcal{P}_{\gamma}^{1}
$$

(The expression for $\omega_{\gamma}(J, L)$ is constant in $\left.t.\right)$

3. We have the decomposition $\mathcal{P}_{\gamma}^{1}=\mathcal{T}_{\gamma} \oplus \mathcal{N}_{\gamma}^{1}$, where $\mathcal{N}_{\gamma}^{1}$ is the $\omega_{\gamma}$-complement of $\mathcal{T}_{\gamma}$. In fact, $\mathcal{N}_{\gamma}^{1}$ is given by

$$
\mathcal{N}_{\gamma}^{1}=\left\{J \in \mathcal{P}_{\gamma}^{1}: \mathbf{g}(J(t), \dot{\gamma}(t))=0 \text { for every } t \in[0, T]\right\}
$$

That is, $\mathcal{N}_{\gamma}^{1}$ is the space of nonholonomic Jacobi fields normal to $\dot{\gamma}$.

In the general case, the existence question remains open for further investigation.

\section{References}

[1] Agrachev, A.A., Barilari, D., Boscain, U.: A comprehensive introduction to sub-Riemannian geometry. Cambridge University Press. Cambridge (2020).

[2] Agrachev, A.A., Gamkrelidze, R.V.: Feedback-invariant optimal control theory and differential geometry-I. Regular extremals. J. Dyn. Control Sys. 3, 343-389 (1997).

[3] Agrachev, A.A., Gamkrelidze, R.V.: Feedback-invariant optimal control theory and differential geometry-II. Jacobi curves for singular extremals. J. Dyn. Control Sys. 4, 583-604 (1998).

[4] Barilari, D., Rizzi, L.: On Jacobi fields and a canonical connection in sub-Riemannian geometry. Arch. Math. (Brno) 53, 77-92 (2017). 
[5] Barrett, D.I., Remsing, C.C.: A note on flat nonholonomic Riemannian structures on three-dimensional Lie groups. Beitr. Algebra Geom. 60, 419-436 (2019).

[6] Barrett, D.I., Remsing, C.C.: On geodesic invariance and curvature in nonholonomic Riemannian geometry. Publ. Math. Debrecen 94, 197-213 (2019).

[7] Barrett, D.I., Remsing, C.C.: On the Schouten and Wagner curvature tensors. Preprint.

[8] Barrett, D.I., Biggs, R., Remsing, C.C., Rossi, O.: Invariant nonholonomic Riemannian structures on three-dimensional Lie groups. J. Geom. Mech. 8, 139-167 (2016).

[9] Bellaïche, A., Risler, J-J. (eds): Sub-Riemannian geometry. Birkhäuser. Basel (1996).

[10] Bloch, A.M.: Nonholonomic mechanics and control (2nd ed.). Springer. New York (2003).

[11] Cantrijn, F., Langerock, B.: Generalised connections over a vector bundle map. Differential Geom. Appl. 18, 295-317 (2003).

[12] Cortés Monforte, J.: Geometric, control and numerical aspects of nonholonomic systems. Springer. Berlin (2002).

[13] Dragović, V., Gajić, B.: The Wagner curvature tensor in nonholonomic mechanics. Regul. Chaotic Dyn. 8, 105-123 (2003).

[14] Ehlers,K.: Geometric equivalence on nonholonomic three-manifolds. In: Proceedings of the Fourth International Conference on Dynamical Systems and Differential Equations, Wilmington NC, USA. 246-255 (2002)

[15] Koiller, J., Rodrigues, P.R., Pitanga, P.: Non-holonomic connections following Élie Cartan. An. Acad. Bras. Cienc. 73, 165-190 (2001).

[16] Langerock,B.: Nonholonomic mechanics and connections over a bundle map. J. Phys. A: Math. Gen. 34, L609-L615 (2001).

[17] Lewis, A.D.: Affine connections and distributions with applications to nonholonomic mechanics. Rep. Math. Phys. 42, 135-164 (1998).

[18] Michor, P.W.: Topics in differential geometry. American Mathematical Society. Providence, RI (2008).

[19] Montgomery, R.: A tour of subRiemannian geometries, their geodesics and applications. American Mathematical Society. Providence, RI (2002).

[20] Rifford, L.: Sub-Riemannian geometry and optimal transport. Springer. Berlin (2014).

[21] Tavares, J.N.: About Cartan geometrization of non-holonomic mechanics. J. Geom. Phys. 45, 1-23 (2003).

[22] Vershik, A.M., Gershkovich, V. Ya.: Nonholonomic problems and the theory of distributions. Acta Appl. Math. 12, 181-209 (1988).

[23] Vershik, A.M., Gershkovich, V.Ya.: Nonholonomic dynamical systems, geometry of distributions and variational problems. In: Dynamical Systems VII. Springer. Berlin (1994).

\section{Affiliations}

\section{D.I. BARRETT}

ADDRESS: Business Science Corporation, 2196, Sandton, Johannesburg-South Africa.

E-MAIL: dbarrett6@gmail.com

ORCID ID: 0000-0002-2071-1315

\section{C.C. REMSING}

ADDRESS: Rhodes University, Dept. of Mathematics, 6140, Grahamstown/Makhanda-South Africa.

E-MAIL: c.c.remsing@ru.ac.za

ORCID ID: 0000-0002-3972-2591 